\title{
Vorstellungen der Renaissance vom Sitz der Regierung im antiken Rom
}

\section{Hubertus Günther (Universität Zürich)}

Zu Beginn der Renaissance, im Jahr 1446, stellt Flavio Biondo fest, daß der Senat und die Comitia, die Volkswahlen, das Fundament der römischen Republik und der Freiheit bildeten. ${ }^{1}$ Aus den antiken Schriften war bekannt, daß die Republik die Staatsform des römischen Reiches war und bis zum Ende seines Bestehens, also auch unter den Kaisern, blieb. Die Comitia, in denen das Volk die Magistrate wählte, bestanden unter den Kaisern fort. Der Senat war die höchste Regierungsinstanz Roms und blieb es zumindest theoretisch unter den Kaisern. Er erließ die Gesetze, er war verantwortlich für die Außenpolitik und für Krieg und Frieden, er wachte über Ordnung und Moral und den traditionellen religiösen Kult, er nahm teilweise an dauerhaften Gerichten teil. Die Exekutivgewalt lag bei den Magistraten. "Welches die Gebäude sind, wo die Vornehmen ihr Amt ausüben, ist klar: der Senat in der Kurie, der Richter in der Basilika oder im Praetorium, der Feldherr im Feldlager oder in der Flotte, der Priester im Tempel". Das erklärt Leon Battista Alberti in seinem Architekturtrakat (1485 publiziert, aber schon 1451 teilweise vollendet). ${ }^{2}$ Die Kurie wurde sprachlich in Parallele zum Rathaus gesetzt, obwohl sich das Rathaus mit seiner besonderen multifunktionalen Bestimmung erst im Mittelalter entwickelt hat. ${ }^{3}$ Im Mittelalter und in der Renaissance wurde der Begriff curia wie heute noch gewöhnlich mit 'Rathaus', 'Palazzo della Ragione' oder ähnlich übersetzt und umgekehrt das Rathaus oft als curia bezeichnet. ${ }^{4}$ Gelegentlich nannte man das Rathaus auch Praetorium oder Basilica (so Palladios 'Basilica', die freilich nur einen Teil der Ratsbauten von Vicenza, den großen Ratssaal, bildete ${ }^{5}$.

Wir konzentrieren hier die Frage, wie man sich den antiken Regierungssitz in der Renaissance vorstellte, hauptsächlich auf die Kurien. Die Vorstellungen der Renaissance waren durchaus nicht einheitlich und nicht immer wirklich konkret. Hier wird nicht die ganze Vielfalt an Varianten berücksichtigt. Es geht nur darum, die wichtigsten Ideen ins Auge zu fassen, sie zu beschreiben und ihren geistigen Hintergrund zu beleuchten.

\section{Rom, Regierungssitz eines Weltreiches}

Man sollte denken, daß die Regierung eines Weltreiches, wie es Rom war, einen großartigen Sitz hatte. Im Anschluß an Aristoteles und andere antike Autoren behandeln zahllose Schriften der Renaissance die Bedeutung der Magnificentia. ${ }^{6}$ Es geht letztlich um die Binsenweisheit, die heute so gut wie damals gilt, daß die Erscheinung der Architektur

${ }^{1}$ F. Biondo, Roma instaurata, II 82.

${ }^{2}$ L. B. Alberti, De re aedificatoria V 6. Übers. M. Theuer, L.B. Alberti, Zehn Bücher über die Baukunst, Wien/Leipzig, 1912, p. 236. Diese Ed. wird hier zitiert; vgl. die lat./ital. Ed. v. G. Orlandi/ P. Portoghesi, Milano, 1966.

3 S. Albrecht, Mittelalterliche Rathäuser in Deutschland. Architektur und Funktion, Darmstadt, 2004; J. Paul, Die mittelalterlichen Kommunalpaläste in Italien, Diss. Freiburg, 1963; M. Borkenstein Neuhaus, Civitas - Vorstellung und Wirklichkeit. Architekturund Urbanistik im mittelalterlichen Italien, Oberhausen, 2001, pp. 77-99.

\footnotetext{
${ }^{4}$ Vgl. neben E. Forcellinis Lexicon totius latinitatis die Lexika des mittelalterlichen Latein, wie bes. Du Cange, Glossarium mediae et infimae latinitatis; Dictionary of Medieval Latin der British Academy (bisher bis Phi); Lexicon latinitatis Nederlandicae Medii Aevi; Lexicon Mediae et infimae latinitatis Polonorum etc.

5 Vgl. den Kommentar dazu in: A. Palladio, Quattro libri dell'architettura, Venezia, 1570, III caput 20.

${ }^{6}$ Vgl. neuerdings K. Imesch, Magnificenza als architektonischeKategorie. IndividuelleSelbstdarstellung versus Verwirklichung von Gemeinschaft in den venezianischen Villen Palladios und Scamozzis, Oberhausen, 2003, pp. 37-68 und passim; dort weitere Literatur.
} 
großartig soll sein, weil so das Ansehen der Bauherrn vermehrt wird, und dies gilt in besonderem Maße für öffentliche Architektur, sicher auch für Bauten mit praktischen Funktionen, doch am meisten für die Regierungssitze mit repräsentativer Funktion. Der Staat demonstriert seine Macht gewissermaßen in der Großartigkeit seiner Regierungsbauten. Alberti hält gleich zu Beginn seines Architekturtraktates fest, ${ }^{7}$ wie wichtig die repräsentative Erscheinung der Architektur für den Staat sei, und bekräftigt das in der Folge immer wieder, am nachhaltigsten im sechsten Buch, das den Schmuck im Allgemeinen behandelt. Die repräsentative Erscheinung sei nötig, sagt er, um die Achtung sowohl der eigenen Bürger als auch ausländischer Regierungen hervorzurufen. Er bespricht zudem den Aspekt, daß die äußere Erscheinung über den dekorativen Wert hinaus konkrete politische Bedeutung hat: Sie dient zur Festigung der Sicherheit und Stärkung der Macht. Das sagt er generell und weist bei konkreten Beispielen wie dem Wehrbau auch auf den praktischen Effekt der äußeren Erscheinung hin. ${ }^{8}$ So heißt es beispielsweise: "Ich möchte auch, daß die Mauern einer Stadt derart seien, daß bei ihrem Anblick der Feind erbebt und alsbald kleinmütig abzieht”.

Solche Redensarten drücken nur aus, was man in der Renaissance seit altersher gewohnt war: Leonardo Bruni stellt in seiner Laudatio auf Florenz (um 1403) fest, das Aussehen einer Stadt zeuge generell von ihrer Kraft. Der Palazzo Vecchio, das Rathaus, lasse schon durch seine äußere Erscheinung erkennen, was seine Funktion ist. Sein Rang zeigt sich in Größe, Aufwand und sogar in seinem Stil, denn er nimmt einen befestigten Adelssitz zum Vorbild:

"Mitten unter diesen Gebäuden aber ragt der gewaltige und durch seine Schönheit beeindruckende Komplex einer Burg, der Signoria, der allein schon durch sein Äußeres zu erkennen gibt, wozu er errichtet wurde. Wie nämlich in einer großen Flotte das Admiralsschiff durch sein Aussehen erkennen läßt, daß auf ihm der oberste Befehlshaber der Flotte fährt, so ist das Erscheinungsbild der Signoria derart, daß jeder leicht urteilen kann: Hier wohnen die Lenker des Gemeinwesens. So prächtig ist sie erbaut, so steil ragt sie empor, daß sie alle Gebäude in weitem Umkreis beherrscht und ihr mehr als privater Rang deutlich ist".?

Ähnliches läßt sich über die meisten Ratshäuser oder über den Dogenpalast von Venedig sagen. Sie bilden das bürgerliche Wahrzeichen ihrer Stadt. Das ist natürlich auch heute noch allgemein bekannt. Es wird hier nur angeführt, um daran zu erinnern, vor welchem Hintergrund die Vorstellungen der Renaissance vom antiken Regierungssitz in Rom entstanden.

Man war sich zu Beginn der Renaissance bewußt, daß die antike Zivilisation die eigene weit übertraf und daß die alten Römer über ungleich großartigere Mittel verfügten als die kleinen italienischen Stadtstaaten. Das Zentrum des einstigen Weltreichs übertraf alles auf der Welt, war die allgemeine Meinung. Alberti kleidete sie in die Worte:

\footnotetext{
7 Alberti, De re aed. Vorrede. Theuer, p. $12 \mathrm{ff}$.

8 De re aed. VI 2, VII 2. Theuer, pp. 293, 347. Wdht. z.B. von G. Philandrier, In Decem Libros $M$. Vitruvii Pollionis De Architectura Annotationes, Roma, 1544. Vgl. J. Białostocki, 'The power of Beauty. A utopian idea of Leone Battista Alberti', in: Studien zur toskanischen Kunst. Festschrift für L.H. Heydenreich, München, 1964, pp. 13-19.
}

\footnotetext{
9 H. Baron, From Petrarch to Leonardo Bruni. Studies in Humanistic and Political Literature, Chicago/ London, 1968, pp. 232-263; N. Mout (ed.), Die Kultur des Humanismus. Reden, Briefe, Traktate, Gespräche von Petrarca bis Kepler, München, 1998, pp. 43-55.
} 
“... so schien die Kunst ihre ganze Kraft darein zu setzen, daß das Weltreich, welches alle anderen Vorzüge auszeichneten, auch durch ihren Schmuck um so bewunderungswürdiger werde. Also ließ sie sich vollständig erkennen und in Besitz nehmen, weil sie es natürlich als eine Schande betrachtete, wenn die Hochburg des Erdkreises und die Zierde der Menschheit im Ruhm ihrer Bauwerke von jenen erreicht würde, welche sie in allen anderen Vorzügen rühmlichst überragte". ${ }^{10}$

Daß diese Vorstellung angebracht war, bezeugten in den Augen der Renaissance die riesigen Theater, Zirkusse, Thermen, oder der Kaiserpalast auf dem Palatin, obwohl er inzwischen weitgehend zerstört war, allein schon durch seine gigantischen Substruktionen und auch die riesigen Anlagen, die man für Privathäuser der Vornehmen hielt, also derjenigen, aus denen sich der Senat zusammensetzte. Selbst bei den Bauten, die dem reinen Vergnügen dienten, ließ sich beobachten, daß die großartige Erscheinung eine fruchtbare politische Wirkung ausübte. Tacitus berichtet davon, wie eine germanische Delegation, als sie ins Pompeiustheater geführt wurde, so beeindruckt von dessen enormer Größe und Pracht war, daß sie spontan die Römer als die machtvollste Nation der Welt pries und sich bereitwillig als ihre Bundesgenossen erklärte. ${ }^{11}$ Der Bericht war in der Renaissance berühmt. Biondo zitiert ihn bereits. ${ }^{12}$ Alberti preist das Pompeiustheater demgemäß als würdig der "Siegerin Rom" ${ }^{13}$ Ebenso bekannt war die Angabe Varros, daß die Kurie eigens dazu bestimmt war, Gesandte zu empfangen, die fremde Völker zum Senat geschickt hatten. ${ }^{14}$ So lag es von vornherein nahe anzunehmen, daß sie großartig aussah, und Vitruv bestätigt das mit dem allergrößtem Nachdruck: "Besonders muß gerade die Kurie so gemacht werden, daß sie vor allem der Würde der Stadt oder des Staates entspricht". 15 Große Ausmaße mußte die Kurie wohl allein schon deshalb haben, weil der Senat ein großes Gremium war. Er hatte gewöhnlich dreihundert Mitglieder, zeitweise sechshundert und sogar tausend. ${ }^{16}$

\section{Das Kapitol}

Bis zum Beginn der Renaissance war allgemein die Vorstellung verbreitet, daß der Sitz der römischen Regierung märchenhaft grandios war. Diese Vorstellung verband sich noch nicht konkret mit einer Kurie, sondern war vage auf das Kapitol bezogen, das in den antiken Schriften als Garant der römischen Weltherrschaft erscheint und als eindrucksvollstes Monument der Welt hingestellt wird. ${ }^{17}$ Im Mittelalter bildete sich die Überzeugung, der Senat habe auf dem Kapitol residiert. Die Mirabilien geben seit dem 12. bis ins 15. Jahrhundert an: die Konsuln und Senatoren hätten sich auf dem Kapitol versammelt, um die Welt zu regieren, und der Name "Capitolium" wurde von "caput mundi", Haupt der Welt, abgeleitet. ${ }^{18}$

Im 12. Jahrhundert richteten sich die Konservatoren von Rom mit dem Senator an ihrer Spitze wirklich auf dem Kapitol ein. ${ }^{19}$ Aber dieses Gremium war weit davon entfernt, die

\footnotetext{
10 De re aed. VI 3. Theuer, p. 298.

11 Tacitus, Annales XIII 54.

12 F. Biondo, De Roma triumphante libri decem, Venezia, 1511, p. 41v.

13 De re aed. II 2. Theuer, p. 73.

14 Varro, Ling.lat. V 155.

15 Vitruv V 2.

${ }^{16}$ Cassius Dio 40.50.2; Cicero, fin. 5.1.2; Sueton, Aug. 35.

17 Ammianus Marcellinus 22.16.12. C. Edwards, Writing Rome. Textual approaches to the city, Cambridge, 1996, p. $34 \mathrm{ff}$ u. allg. zum Kapitol: pp. 34-41, 71-95.
}

\footnotetext{
18 Codice Topografico della Città di Roma. Ed. R. Valentini/ G. Zucchetti, Roma, 1940-1953, III, pp. 51, 120, 192; IV, p. 140 (Anon. Magl. ca. 1411), 416 (Gio. Rucellai, 1450) etc. Ebenso die Mirabilien des 15. Jh.s in Nationalsprachen. A. Graf, Roma nella memoria e nelle immaginazioni del Medio Evo, Rom/ Florenz, 1882-83, vol. I, p. 184ff; N. R. Miedema, Die "Mirabilia Romae". Untersuchungen zu ibrer Überlieferung mit Edition der deutschen und niederländischen Texte, Tübingen, 1996, p. 348.

19 H. Siebenhüner, Das Kapitol in Rom: Idee und Gestalt, München, 1954; R. Krautheimer, Rome. Profile of a city, Princeton, 1980.
} 
Welt zu regieren. Es hatte nur administrative Aufgaben und Befugnisse; die Regierungsgewalt lag ausschließlich beim Papst. Die Stadt Rom hatte ihre Macht verloren. Rom, einst das Haupt der Welt, spottet Boccaccio, war zum Schwanz der Welt mutiert: "la quale come oggi è coda così già fu capo del mondo ..." ${ }^{20}$ Dieser Spruch ging weit um. ${ }^{21}$ Das Kapitol hatte seinen alten Glanz verloren. Es war großteils von Gebüsch bewachsen, Ziegen klettern darauf herum; Monte Caprino nannte man es deshalb. Die Tempel, die dort einst standen, waren längst zerstört. Nur verstreute Trümmer und einige Teile der alten Substruktionen des Hügels waren noch erhalten. Am Abhang über dem Forum Romanum tritt das Tabularium bis heute in Erscheinung, ausgezeichnet durch eine anspruchsvolle Disposition von Arkaden zwischen einer Blendgliederung von Halbsäulen im Prinzip ähnlich wie am Marcellustheater oder am Kolosseum. Giovanni Francesco Poggio Bracciolini nimmt in seiner Abhandlung De varietate fortunae (1431-48) Rom als Paradigma für den Wandel des Glücks. Er steigt auf das Kapitol, für ihn Zentrum und Regierungssitz des alten Rom, um von dort oben den Blick über die verfallene Stadt schweifen zu lassen. Das Kapitol, klagt er, einst Haupt des Römischen Reiches und Burg der ganzen Welt, sei desolat und zugrunde gerichtet. Der frühere Glanz sei in eine Halde von Abfall und Dreck verwandelt, Unkraut wuchere zwischen den Sesseln der Senatoren. ${ }^{22}$

Im Mittelalter stellte man sich vor, auf dem Kapitolinischen Hügel habe ein herrliches Schloß aufgeragt. Diese Vorstellung erwuchs aus der Überlieferung von der berühmten Burg (arx), die einst dort stand; im übrigen war sie eine reine Fiktion. Manche glaubten, der Senat habe unter dem Schloß residiert, so daß kein Ton von dem, was beraten wurde, nach außen habe dringen können. Das Libro imperiale (um 1400, mehrfach in der Renaissance abgeschrieben und im Druck ediert) phantasiert von einem rundem Raum unter dem Rathaus; sein Boden und die Sitze der Senatoren seien ganz aus Porphyr gewesen, Mosaiken hätten die Decke geschmückt, und an den Wänden hätten rundum Götterstatuen gestanden. ${ }^{23}$ Dieser Ratsaal wurde im 15. Jahrhundert anscheinend mit dem Tabularium assoziiert. ${ }^{24}$ Man stellte sich den Palast auf dem Kapitol so prächtig vor, wie es dem Haupt der Welt gebührt, ${ }^{25}$ als erstes aller Weltwunder, wie es der Pseudo-Beda nennt. ${ }^{26}$ Im Laufe des Mittelalters wurden die Vorstellungen von der alten Erscheinung des Kapitols zunehmend phantastischer. Die Mirabilien in vielen Landessprachen beschreiben es als Palast oder Burg über hohen und sicheren Mauern, das als ein Spiegel der ganzen Welt erschienen und so kostbar wie der dritte Teil der Welt gewesen sei. Es sei über und über mit vergoldetem Erz und Edelsteinen geschmückt und mit Kristall gedeckt gewesen. Die Halle des Schlosses habe einen Boden von Erz und eine Decke aus Kristall gehabt. Mit ähnlichen Metaphern für die glanzvollste Erscheinung, die man sich nur denken kann, beschreibt Albrecht von Scharffenberg im Jüngeren Titurel die Gralsburg. Über der Decke des Ratssaals imaginieren die deutschen Mirabilien noch einen Teich, so daß, wie sie versichern, die Senatoren, wenn sie nach oben schauten, über sich die Fische im Wasser schwimmen sehen konnten. In dieser Halle soll die "Salvatio Romae" gestanden haben, eine fabelhafte Gruppe von Statuen aus Erz, die alle

\footnotetext{
20 Boccaccio, Decamerone 5.3.4.

${ }^{21}$ Sogar vom spanischen Pilger Pero Tafur in 1436 paraphrasiert. M. Vaquero Pineiro, Viaggiatori spagnoli a Roma nel Rinascimento, Bologna, 2001, p. 47.

${ }^{22}$ Poggio Bracciolini, De varietate fortunae. Ed. Pekka Suvanto, Helsinki (Annales Academiae scientiarum fennicae, Ser. B, Vol. 265), 1993, p. 98.

${ }^{23}$ A. Graf, Roma nella memoria e nella immaginazione del medioevo, Turin, 1923, p. 217.
}

\footnotetext{
24 Pero Tafur, s. Vaquero Pineiro 2001 (note 21), 42ff; Antiquarie prospetiche Romane. Ed. G. Agosti/D. Isella, O.O., 2004, p. 20.

25 Graf 1882-83 I (note 18), pp. 182-213; Miedema 1996 (note 18), pp. 348ff, 421-425.

${ }^{26}$ M. Demus-Quatember, 'Zur Weltwunderliste des Pseudo-Beda und ihren Beziehungen zu Rom', Römische Historische Mitteilungen 12, 1968-1969, pp. 67-92; Graf 1882-83 I (note 18), p. 185.
} 


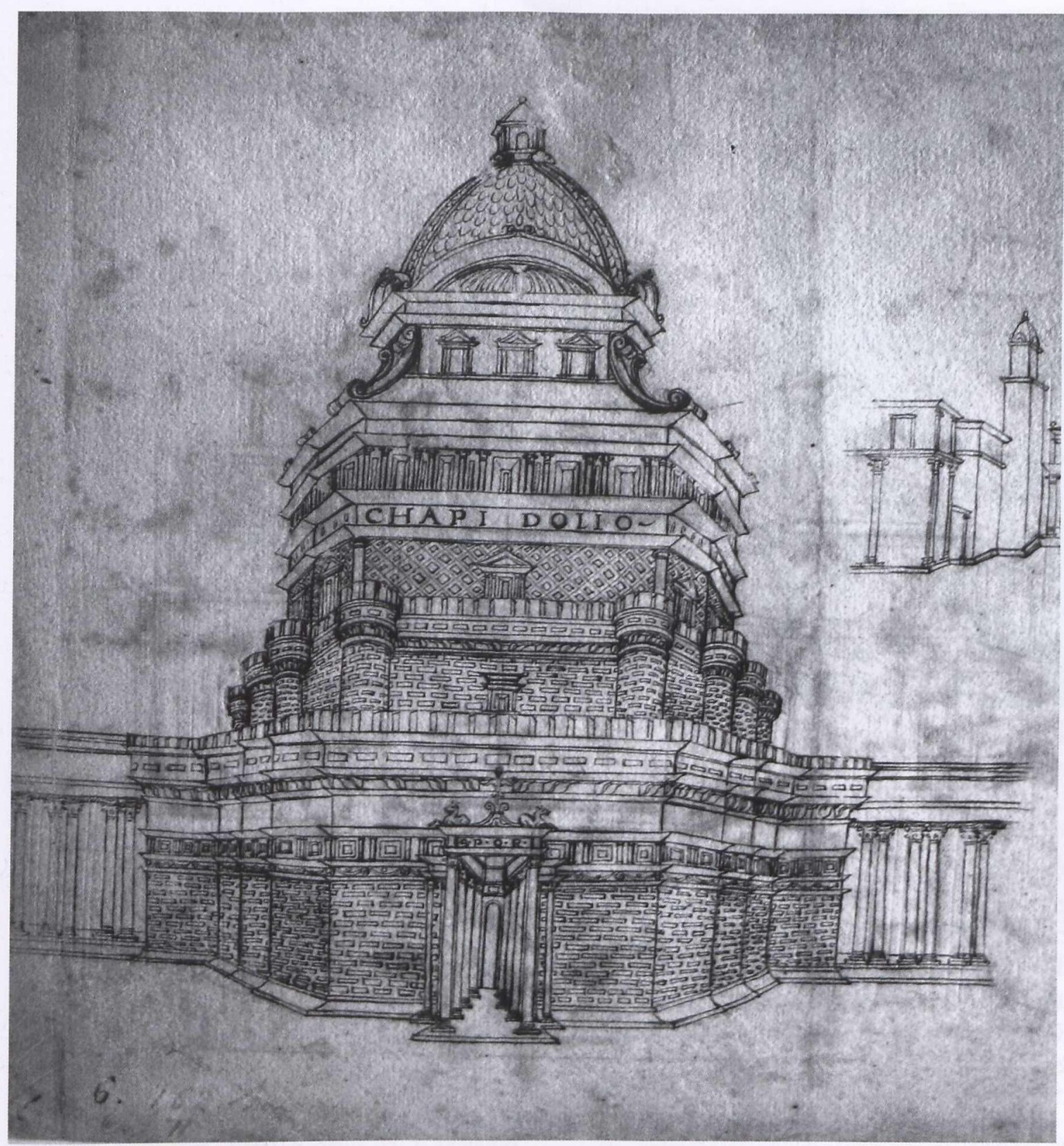

1 Das Kapitol zu Rom, anon. Zeichnung, um 1500. Uffizi, Gabinetto dei Disegni, Sant. 156 (Arte Lombarda 15-II, 1970).

Provinzen des Römischen Reichs darstellten und auf wunderbare Weise anzeigten, wenn in einer Region Gefahr drohte. ${ }^{27}$

Ein besonders suggestives Beispiel für die bildliche Darstellung der Vision vom antiken Kapitol befindet sich in einer Gruppe italienischer Zeichnungen von Phantasiebauten, die um 1500 entstand und sich auf toskanische bzw. sienesische, lombardische und m.E. auch flämische Vorlagen stützt (fig. 1). ${ }^{28}$ Die früheste bekannte Version wurde um

27 Demus-Quatember 1968-1969 (note 26), p. 72ff;

D. Comparetti, Virgilio nel Medioevo, Firenze, 1981

(1. Ed. 1872), II, pp. 70-74; Graf 1882-83 I (note 18), pp. 188-213; Miedema 1996 (note 18), p. $426 \mathrm{ff}$.
28 Uffizi, Gabinetto dei Disegni, Sant. 157-166. A. Bartoli, I monumenti antichi di Roma nei disegni degli Uffizi di Firenze I, Roma, 1914, p. 8ff, Nr. 23-42; G. Scaglia, 'Fantasy architecture of Roma antica', 


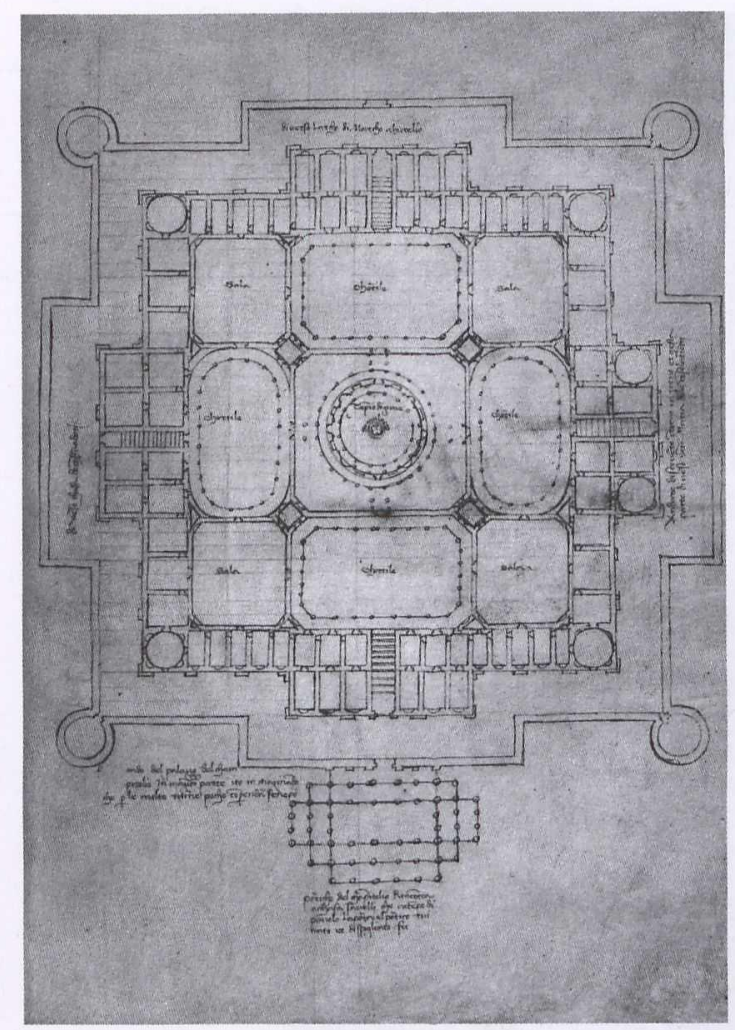

2 Das Kapitol nach Francesco di Giorgio Martini. Cod.Tor.Sal. 148, fol. 82r. (Ed. Maltese 1967, pl. 151).
1460/70 von Buonaccorso Ghiberti gezeichnet, aber in der Folge wurde die Serie anscheinend erweitert und ausgeschmückt. Die derart bereicherte Version von ca. 1500 war im 16. Jahrhundert höchst beliebt. Sie wurde wohl mehr als alle anderen Phantasiedarstellungen der Renaissance kopiert.

Das Kapitol ist hier als hoch aufragender Bau dargestellt, der alle Elemente von Größe, Würde, Schönheit und Pracht vereint. Sein Grundriß bildet ein gleichmäßiges Polygon mit sechzehn Ecken, dessen vier Hauptseiten weit vorkragen. Das Erdgeschoß gehört offenbar zur Befestigung des Kapitolinischen Hügels; darauf steht die Burg, die Arx der antiken Schriften, charakterisiert durch die typischen Elemente mittelalterlicher Kastelle, mit Ecktürmen, Zinnen und Rustizierung der Mauer. Auf diesem Sockel erhebt sich der Palast der Senatoren, ein reiner Idealbau ohne Parallelen in der realen Architektur. Um seine Großartigkeit vor Augen zu führen, sind die vornehmsten architektonischen Elemente eingesetzt, wie die bekrönende Kuppel und die Inkrustation des Erdgeschosses in der Art der Fassaden des Dogenpalastes in Venedig, damals wohl dem prächtigsten öffentlichen Profanbau in Italien und Sitz einer der wenigen Senate in Italien, die wirklich Macht bewahrt hatten. Der Bau ist offenbar einbezogen in eine weite systematisierte urbane Zone, denn von den vier Hauptseiten des Unterbaus gehen große Säulenhallen aus.

Diese Vision findet ein gewisses Gegenstück in der 'Rekonstruktion' des antiken Kapitols, die Francesco di Giorgio um 1480 im Grundriß mit Aufriß des Eingangsportikus gezeichnet hat (fig. 2) ${ }^{29}$ Francesco räumt allerdings ein, daß er seine 'Rekonstruktion' zum größten Teil frei imaginiert habe. Auf dem in regelmäßiger Form befestigten Sockel des Kapitols steht ein Palast von immenser Ausdehnung, der zwischen gewaltigen Sälen und Höfen in der Mitte den Tempel des Jupiter, einen runden Peripteros, einschließt. Realiter bietet der Kapitolinische Hügel für eine derart weite Anlage sicher nicht genügend Platz.

\section{Die antike Kurie und Antikenforschung im 15. Jahrhundert}

Derartige Vorstellungen, die auf freier Phantasie beruhen, sind nicht typisch für den Rückblick der Renaissance auf die Antike. Wenn wir versuchen, auf Grund von Beschreibungen zu erfassen, wie man sich in der Renaissance die antike Kurie vorstellte,

Arte Lombarda 15, 1970, Nr. 2, pp. 9-24; T. Buddensieg, 'Criticism of ancient architecture in the sixteenth and seventeenth centuries', in: R. R. Bolgar (ed.), Classical Influences on European Culture, A.D. 1500-1700, Cambridge, 1976, pp. 335-348, spez. p. 339; C.L.Frommel, 'Peruzzis römische Anfänge. Von der "Pseudo-Cronaca-Gruppe" zu Bramante',
Römisches Jabrbuch der Bibliotheca Hertziana 27-28, 1991-1992, pp. 137-182, spez. p. 139ff.

${ }_{29}$ Cod. Tor. Saluzz. 148, f. 81v.-82r. Francesco di Giorgio, Trattati di architettura ingegneria e arte militare. Ed. C. Maltese, L. Maltese Degrassi, Milano, 1967, Taf. $150 f f$. 
so ergibt sich ein enttäuschendes Ergebnis. Wir sind dann im wesentlichen, wie so oft, auf Albertis Architekturtraktat angewiesen. Alberti beschreibt Bautypen, die für die Neuzeit relevant sein sollen, aber realiter meist antike Bauten zum Vorbild nehmen und die moderne Baupraxis kaum berücksichtigen. Beispiele dafür bilden die Thermen, Theater, Mausoleen, Triumphbögen etc. Für die Kurie gilt das gleiche. Wir werden darauf noch genauer eingehen. Vorab stellen wir nur fest, daß Alberti die Kurie einfach als einen Saal beschreibt (fig. 6-7). ${ }^{30}$ Dieser Saal hat eine gewisse architektonische Gliederung, aber sonst nichts. Seine Dimensionen sind nicht angegeben, aber nach den praktischen Gliedern, Tür und Fenstern, ist er nicht auffallend groß. Er steht jedenfalls in keinem Verhältnis zur Größe des Römischen Reichs.

Wir können aber auch einen anderen Weg einschlagen. Er ist allerdings etwas mühsam. Wir müssen uns dann nämlich darauf einlassen, die für die Renaissance typischen wissenschaftlichen Untersuchungen mit ihrem ganzen positivistischen Vorgehen nachzuvollziehen. ${ }^{31}$ Diese Mühe lohnt siç indessen. Nebenbei ist sie auch aus wissenschaftsgeschichtlicher Sicht ergiebig, denn die Renaissance hat, indem sie die induktive Methode zur Grundlage ihrer Untersuchungen machte, den Weg der modernen Wissenschaft eröffnet. Am Anfang dieser Entwicklung stehen die Forschungen zur Antike. Die Antiquare gingen im Prinzip ebenso rational vor, wie es die Archäologen bis heute tun sollten. Im Unterschied zu den einschlägigen Kommentaren aus dem Mittelalter wurde jetzt nicht mehr frei assoziiert, sondern systematisch gesammelt, was an Zeugnissen erhalten war: Hinweise in der antiken Literatur, Inschriften und Ruinen, und dies Material wurde dann präzise ausgewertet. Es ist bekannt, wie gründlich Alberti, um zu seinen Angaben über das Aussehen der antiken Bautypen zu gelangen, die Ruinen untersuchte, und wie viele Schriften er verarbeitete. Biondo, einer der Protagonisten der modernen Archäologie, behandelt sämtliche wichtigen antiken Bauten der Stadt in seinen grundlegenden Werken über das antike Rom: der Beschreibung des alten Rom, De Roma instaurata (publ. 1446), ${ }^{32}$ und der römischen Kulturgeschichte, Roma triumphans (vollendet 1459). ${ }^{33}$ Dafür stützt er sich ausdrücklich auf mehr als dreihundert antike Schriften, das ist fast der gesamte Bestand an Literatur, der bis heute für die römische Archäologie zur Verfügung steht. ${ }^{34}$ Poggio und dann viele andere Humanisten sammelten alle erreichbaren Inschriften. Auch diese Sammlungen sind bis heute grundlegend geblieben, obwohl sie im Lauf der Zeit ergänzt wurden.

\footnotetext{
30 De re aed. VIII 9.

31 R. Weiss, The Renaissance discovery of classical antiquity, New York, 1988²; S. Settis (ed.), Memoria dell'Antico nell'Arte italiana, Torino, 1984-1986; M. Fagiolo (ed.), Roma e l'Antico nell'Arte e nella Cultura del Cinquecento, Roma, 1985; H. Günther, Die Renaissance der Antike, Weimar, 1998.

32 Valentini/ Zucchetti IV (note 18), pp. 247-324; D. M. Robathan, 'Flavio Biondo's "Roma instaurata" ', Medievalia et Humanistica, N.S. I, 1970, pp. 203-216; A. M. Brizzolara, 'La Roma instaurata di Flavio Biondo: Alle origini del metodo archeologico', Atti della Accademia delle Scienze dell'Istituto di Bologna, Cl. scienze mor., Memorie LXXVI, 1979-1980, pp. 29-74; O. Clavuot, Biondos "Italia illustrata" - Summa oder Neuschöpfung?, Tübingen, 1990; H. Günther, 'L'Idea di Roma antica nella "Roma instaurata" di Flavio Biondo', in:
}

S. Rossi (ed.), Le due Rome del Quattrocento, Roma, 1997, pp. 380-393.

33 A. Mazzocco, 'Some philological aspects of Biondo Flavio's Roma triumphans', Humanistica Lovaniensia 28, 1979, p. 12ff; M. Tomassini, C. Bonavigo, Tra Romagna ed Emilia nell'Umanesimo, Bologna, 1985, pp. 9-80. Rezension von R. Fubini, Roma nel Rinascimento, 1987, p. 174ff; L. Capra, "Un tratto di "Roma triumphans" omesso dagli stampatori', Italia Medioevale e Umanistica 20, 1977, pp. 303-322.

${ }^{34}$ Mazzocco 1979 (note 33), p. 18ff; Tomassini/ Bonavigo 1985 (note 33), p. 79ff; Brizzolara 1979-1980 (note 32), p. 32ff; P. Buchholz, Die Quellen der Historiarum decades des Flavius Blondus, Diss. Leipzig, 1881. 


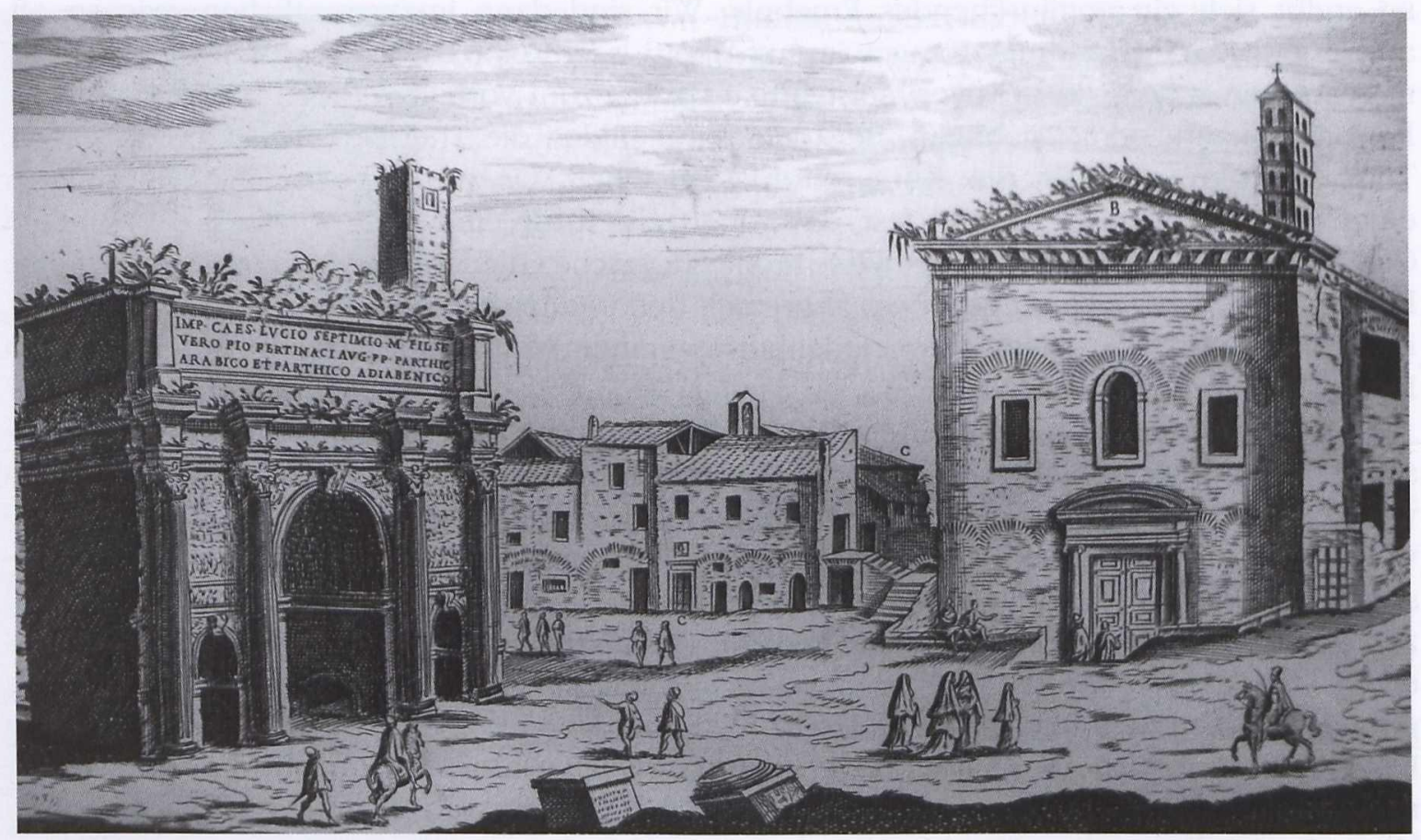

3 Zone des Forum Romanum unterhalb vom Kapitol, mit rechts S. Adriano (nach heutigem Verständnis ehemals die Curia Julia, errichtet an der Stelle der Curia Hostilia). S. Dupérac, Vestigi di Antichità, 1575, pl. 3 (Ed. Losi, Roma, 1773). Universiteit Utrecht.

Die Vorstellung, daß das Kapitol einst Sitz der römischen Regierung war, findet keinerlei Bestätigung in den antiken Schriften. Trotzdem ist sie bis heute populär. Davon zeugt etwa die Bezeichnung 'Kapitol' für das Regierungszentrum der USA, den Sitz von Kongreß und Senat in Washington. So ist es nicht verwunderlich, daß auch bei Humanisten wie Poggio die Idee vom Kapitol als Regierungssitz nachwirkte. Jean Poldo d'Albenas gebraucht noch in seinem Buch über das antike Nîmes (1559) den Ausdruck 'Kapitol' synonym mit Kurie oder Sitz der Regierung. Er hält die sogenannte Maison Carrée, also einen Antentempel, für die "maison Consulaire" des alten Nîmes. ${ }^{35}$ Es gab immerhin einige konkrete Anhaltpunkte dafür, das Kapitol mit dem antiken Sitz der Regierung zu assoziieren. Einer davon war die Nähe zum Forum Romanum. Diverse antike Schriften geben an, daß die Curia Hostilia zusammen mit der Rostra, dem Comitium etc. beim Forum gelegen habe. Das ist einer der Gründe für die heutige Lokalisierung dieser Stätten (fig. 3). ${ }^{36}$ Poggio entdeckte eine Inschrift aus der Zeit des Kaisers Theodosius, aus der sich ergibt, wo das Senatulum (Secretarium Senatus) lag, ${ }^{37}$ das aus antiken Schriften bekannt war und in dem sich nach Varro ${ }^{38}$ der Senat versammelte. Es befand sich am Forum unterhalb des Kapitols, ganz nahe beim vermeintlichen Schatzhaus (S. Adriano). Der antike Bau war damals in der Kirche von S. Martina sogar noch bewahrt (von Pietro da Cortona erneuert, SS. Luca e Martina). Flavio Biondo und viele Spätere folgten Poggios Lokalisierung des Senatulum. ${ }^{39}$ Überdies war überliefert, daß die Richtstätte am

35 Poldo d'Albenas, Discours historial de l'antique et illustre cité de Nismes, Lyon, 1559, pp. 73-78.

${ }^{36} \mathrm{Vgl}$. außer den einschlägigen topographischen Lexica Roms auch den praktischen, wenn auch stark kompilatorischen Überblick von D. Gneisz, Das antike Rathaus, das griechische Bouleuterion und die frübrömische Curia, Wien, 1990. Besprechung von H. Lauter in Gnomon 63, 1991, p. 745ff.

37 CIL VI, p. XXXVII nr. 63 (=nr. 1718); Poggio, De varietate fortunae; Valentini/Zucchetti IV (note 18), p. 242.

38 Varro, Ling.lat. V 156.

39 Biondo, Roma inst. II 65. 
Tarpeischen Felsen lag. In den Illustrationen der Sylloge des Giovanni Marcanova (Padua, ca. 1465) ist das Kapitol als Ort der höchsten weltlichen Gewalt gekennzeichnet, indem es als Tarpea bezeichnet ist und gezeigt wird, wie hier Todesurteile vollstreckt wurden. ${ }^{40}$

\section{Flavio Biondo}

Biondo veränderte die Vorstellungen von den Regierungsbauten gründlich. Er setzt sich mit ihnen sowohl in Roma triumphans, als auch in Roma instaurata auseinander. Er widmet dem Thema drei der zehn Bücher von Roma triumphans (3.-5. Buch). Dort erklärt er in erster Linie die staatlichen Institutionen, Regierungsorgane und die Administration; nur beiläufig, wenn überhąupt, erwähnt er hier, wo sie ihre Sitze hatten. Die Rombeschreibung lieferte bereits eine ausführliche Einführung zu den staatlichen Institutionen, aber hauptsächlich war sie dazu bestimmt, deren Sitze zu identifizieren. Sie bildet keineswegs eine geschlossene Darlegung, sondern konzentriert sich auf einzelne topografische Angaben und streut dazwischen bei manchen Bauten ausführlichere Abhandlungen über ihre Funktion ein. Wenn man besondere Bautypen überblicken will, muß man sich die Angaben zu den Ruinen, die dazugehören, an diversen Stellen zusammensuchen. Biondo gibt die Argumentation für seine Lokalisierungen nur teilweise und knapp an, den Rest muß man sich mit Hilfe der einschlägigen antiken Literatur hinzudenken. Wir werden teilweise die antiken Schriften zitieren, auf die sich Biondo stützt, um einen Eindruck davon zu vermitteln, wie sehr er sich an den Quellen orientierte. Aber wir können seine Argumentation nicht in extenso nachvollziehen, denn es geht hier ja weniger um die Art der wissenschaftlichen Untersuchung, als um die Vorstellungen, die dahinter standen.

Biondo wandte sich erstmals gegen die populäre Meinung, daß das Kapitol Sitz der Regierung gewesen sei und stellte richtig, daß es hauptsächlich sakralen Zwecken diente. Die Regierungsbauten lokalisierte er nun an anderen Orten. Aber auch er war befangen von dem, was er gewohnt war und was zu seiner Zeit selbstverständlich schien. Er ging anscheinend von der Hypothese aus: Die obersten Regierungsgremien hatten feste Hauptsitze und deren Erscheinung entsprach der Würde der Gremien und der Macht des Staates. Er konzentriert sich darauf, den Ort des Comitium im Marsfeld und die beiden wichtigsten Kurien zu lokalisieren. Die meisten antiken Staatsbauten lagen nach Biondo gewissermaßen um das Kolosseum herum, das im Mittelalter oft als das Zentrum Roms bezeichnet wurde: auf der einen Seite Forum Romanum, Kapitol und Palatin, gegenüber auf dem Caelius und auf dem Esquilin die beiden Hauptkurien (fig. 4).

Über die Kurien berichtet Varro direkt im Anschluß an das Comitium: "Es gab zwei Arten von Kurien: nämlich diejenigen, wo die Priester sich um die Angelegenheiten der Götter kümmerten, wie die Curiae veteres, und diejenigen, wo sich der Senat um die Belange der Menschen kümmerte, wie die Curia Hostilia, so genannt, weil sie zuerst vom König Hostilius errichtet wurde". ${ }^{41}$ Biondo zitiert das. In diesen beiden Kurien, gibt er an, seien priesterliche und zivile Rechtsfälle behandelt worden. ${ }^{42}$ Er ist sich bewußt, daß der Senat an vielen verschiedenen Orten und nicht nur in Kurien tagte. ${ }^{43}$ Aber er geht nicht weiter darauf ein; er berücksichtigt nicht einmal die berühmte Kurie des Pompeius, in der Caesar ermordet wurde. Er konzentriert sich auf die Curiae veteres oder auch Curia vetus und auf die Curia Hostilia als die beiden Hauptkurien. Für sie sucht er in Roma instaurata geeignete Plätze. ${ }^{44}$

\footnotetext{
40 C. Huelsen, H. Jordan, Topographie der Stadt Rom im Altertum, Bd. I, Abt. 3, Berlin, 1907, Taf. 4, 17.

41 Varro, Ling.lat. V 155.

${ }^{42}$ Roma inst. II, p. 87.
}

\footnotetext{
43 Roma inst. II, p. 87 etc.

${ }^{44}$ Curia Hostilia: Roma inst. I pp. 77-78; II pp. 62-63;

III 7. Roma triumph. 1544 , p. 19.
} 


\section{Hubertus GÜNTHER}

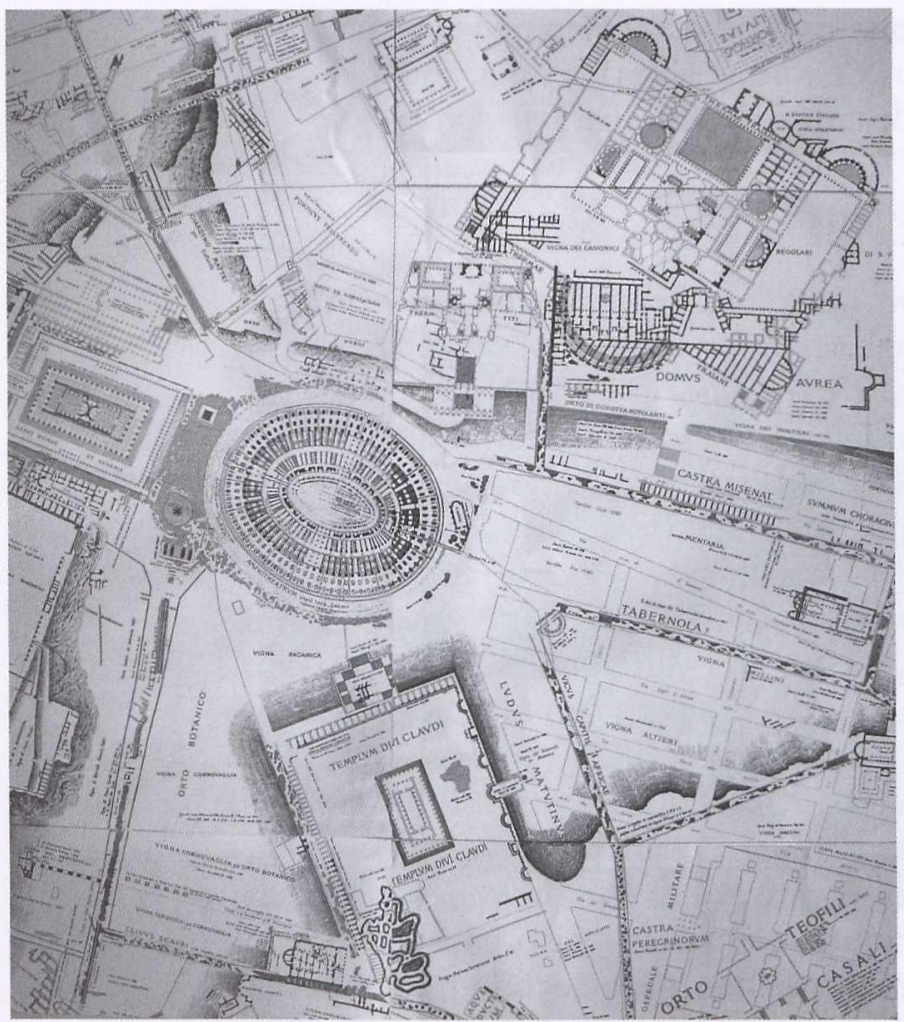

4 Das Kolosseum und Umgebung in der Antike, nach R. Lanciani; links vom Kolosseum das forum romanum, rechts der Mons Caelius mit dem Podest des Claudiustempel, unten (Biondo's Curia Hostilia), oben rechts die Reste der Trajanstermen und der Titustermen (Biondo's Curiae veteres).
Nach Varro hätte es nahegelegen, die Curia Hostilia unterhalb des Kapitols zu lokalisieren, nahe beim Ort des Senatulum, der durch die von Poggio entdeckte Inschrift identifiziertet wurde. Dies ist die allgemeine Meinung der heutigen Archäologen. Aber Biondo folgt Varros Hinweis nicht, vielleicht weil es diverse Senatula gab. ${ }^{45} \mathrm{Er}$ geht von dem Bericht des Livius aus, daß Tullus Hostilius seine Residenz auf dem Caelius errichtete, und dort "einen Tempel zur Kurie machte, die bis auf die Zeit unserer Väter die Hostilische genannt wurde". ${ }^{46}$ Diese Angabe kehrt in den interpolierten Regionenführern unter der Region Caelimontium wieder. ${ }^{47}$ Was die Einrichtung der Kurie in einem Tempel betrifft, so war sie normal und sogar vorgeschrieben. Varro sagt, nur an einem von den Auguren bestimmten Ort dürfe der Senat Beschlüsse fassen, und deshalb seien die Curia Hostilia, die Kurie des Pompeius und die Curia Julia, obwohl sie profane Bestimmung hätten, allesamt von den Auguren bestimmte Tempel. ${ }^{48}$ Biondo zitiert das in einer allgemeinen Abhandlung über die Tempel. ${ }^{49}$ Die Lokalisierung der Curia Hostilia auf dem Caelius wurde noch bestätigt durch die Berichte von den Heiligen Märtyrern Johannes und Paulus, deren Kirche SS. Giovanni e Paolo auf dem Caelius liegt. Die Akten ihres Martyriums geben an, daß sie "iuxta curiam Hostiliam" umgebracht wurden, dies soll

in ihrem Haus geschehen sein, und über diesem Haus soll ihre Kirche errichtet worden sein. ${ }^{50}$ Die Reste der Aqua Claudia, die an die Fassade der Kirche angrenzen, wurden in der Renaissance manchmal als Reste dieses Hauses vorgezeigt. ${ }^{51}$

Für die Curiae veteres findet Biondo einen Ort mit Hilfe mittelalterlicher Tradition. ${ }^{52}$ Aus den antiken Regionenkatalogen von Rom geht hervor, daß die Curiae veteres auf dem Palatin lagen, und weitere antike Schriften bestätigen dies mehr oder minder indirekt. Aber diese Zeugnisse nimmt Biondo nicht wichtig. Vielmehr geht er zunächst von Varros Angabe aus, daß die Auguren über die Via Sacra zu dem Ort gingen, an dem sie ihre Vogelschauen vornahmen, und daß die Via Sacra vom Kapitol zu den "Carinae" führ-

\footnotetext{
45 Festus 519 [Fragment aus Codex Farnese L. XIX].

46 Livius 1, 30, 1-2.

47 Valentini/Zucchetti I (note 18), p. 209.

48 Varro, Epistolicae quaestiones, bei Aulus Gellius Noct. Att. 14, 7, 7.

49 Biondo, Roma triumph. 1544, p. 31v.

50 M. Marchetti, 'Un manoscritto inedito riguardante la topografia di Roma', Bullettino della Commissione Archeologica Communale di Roma 42, 1914 , pp. 41-116, pp. 334-400, spez. 354-355; Acta
}

\begin{abstract}
Sanctorum, Junii, Vol. 5, Antwerpen, 1709, 26. Juni, pp. 158-161; A. M. Colini, Storia e topografia del Celio nell'antichità, Roma, 1944, p. 208; R. Krautheimer, Corpus Basilicarum Christianarum, Città del Vaticano, 1937, p. 268ff; W. Buchowiecki, Handbuch der Kirchen Roms, Wien, 1967-1997, Bd. 2, pp. 126-131. 51 A. Giovannoli, Vedute degli antichi vestigi di Roma, Roma, 1751, Taf. 9.

${ }^{52}$ Roma inst. II pp. 30-35, 38ff, 60; Roma triumph. 1544 , p. 24.
\end{abstract}


te. ${ }^{53}$ Die Region des Esquilin, die zwischen S. Pietro in Vincoli und dem Kolosseum liegt (vulgär genannt "le Capocce"), identifiziert Biondo mit den Carinae, denn, begründet er, so werde sie in alten Rechtsakten und immer noch genannt. Frühere Schriften bestätigen, daß man die Region damals wirklich als Carinae bezeichnete. ${ }^{54}$ Biondo beschreibt nun, wie die Auguren vom Kapitol, durch das Forum Romanum und am Ende durch den Titusbogen zogen, sich dann dem Kolosseum zuwandten und hinter ihm auf den Esquilin zu den Carinae aufstiegen, um dort die Vogelschauen vorzunehmen. Diesen Ort setzt Biondo mit den Curiae veteres gleich. Warum, sagt er nicht. Vielleicht weil er Auguren mit Priestern gleichsetzt und annimmt, daß der Ort, an dem sie ihres Amtes walteten, identisch mit ihrem Hauptsitz sein müßte. Vielleicht auch weil es im damals sehr bekannten Aeneas-Kommentar des Servius heißt, Augustus sei in den Curiae veteres geboren und in den vornehmen Carinae gesäugt worden. ${ }^{55}$

Für beide Kurien weist Biondo nun monumentale Ruinen vor. Mit der Kirche SS. Giovanni e Paolo war ein Palast verbunden, der auf den Fundamenten der vermeintlichen Curia Hostilia liegt. ${ }^{56}$ Diese Fundamente gehören nach heutiger Ansicht zu den Stützmauern des Podiums, auf dem sich der Tempel des Claudius erhob. Sie bildeten die Westfront dieses Podiums. Das Podium umfaßte ein Areal von 180 × $200 \mathrm{~m}$ und war ca. 10 Meter hoch. Von dem Tempel selber hat sich nichts erhalten, doch seine Substruktionen sind teilweise noch heute sichtbar. Sie schlossen im Westen zwei Geschosse ein, die außen im Prinzip ähnlich wie das Tabularium in Erscheinung treten: mit hohen Arkaden und einer Blendgliederung zwischen ihnen. Allerdings bestehen Wand und Gliederung aus grob rustizierten Travertinblöcken. Es ist eines der schönsten antiken Beispiele für die Rustica, die in der Renaissance bekannt waren. Heute sind acht von den Arkaden erhalten; rund hundert Jahre nach Biondo waren es noch achtzehn oder neunzehn von ihnen. ${ }^{57}$ Hinter den Arkaden schließen tonnengewölbte Räume an, deren Mauern aus Ziegelstein bestehen und die an eine mehrschichtige Mauer (über 6 Meter tief) herangebaut sind. Beim heute sichtbaren Rest handelt es sich um das Obergeschoss der Stutzmauern. Das Erdgeschoß ist verschüttet. Die eindrucksvollen Reste sind oft gezeichnet worden in der Renaissance. ${ }^{58}$ Freilich meist nur das obere Geschoß. Piranesi hat beide Geschosse genau aufgenommen. ${ }^{59}$ Etienne Dupéracs Vedute der "Vestigi et parte del Monte Celio" in den Vestigi dell'Antichità di Roma von 1575 zeigt die Front des Podiums fast noch in ihrer gesamten Breite (fig. 5). ${ }^{60}$ Allerdings sind nur jene Arkaden nahe bei SS. Giovanni e Paolo in gutem Erhaltungszustand dargestellt. Die übrigen Fronten waren ähnlich gegliedert. Die Ostfront wurde aber unter Nero umgestaltet mit Nymphäen und den für solche Stützwände typischen Nischen in opus reticulatum. ${ }^{61}$ In der Mitte der Westfront und in der Mitte der Nordfront, die in Richtung Forum wies, führten Freitreppen hinauf zum Tempel. Die Treppe der Nordfront war anscheinend besonders aufwendig. Francesco di

53 Varro, Ling.lat. V 47.

54 Gio. Cavallini, Polistoria de virtutibus et dotibus Romanorum, (um 1350); Anon. Magl. (um 1411); Valentini/Zucchetti IV (note 18), p. 26ff, 126.

55 Servius, Aen. 8.361.

56 Roma inst. I, p. 78.

57 So die Legenden zu Palladios Zeichnungen. G. Zorzi, I disegni delle antichità di Andrea Palladio, Venezia, 1958, Nr. 63-64.

58 So etwa Palladio, loc.cit., u. Gio. Ant. Dosio (Uffizi, Gab. dei Disegni, A 2030, A 2518). G. A. Dosio, Roma antica e $i$ disegni di architettura agli Uffizi, Ed. F.
Borsi, C. Acidini, F. Mannu Pisani, G. Morolli, Roma, 1976, p. $123 \mathrm{ff}$.

59 G. B. Piranesi, Le antichità romane (1756) IV. Idem, Vedute di Roma (1750-1751). Gesamtkatalog der Kupferstiche, Ed. L. Ficacci, Köln etc., 2000, pp. 317, 700. ${ }^{60}$ E. Dupérac, Vestigi dell'antichità di Roma, Roma, 1575, p. 14. J. Garms, Vedute di Roma dal Medioevo all'Ottocento, Napoli, 1995, Bd. 2, p. 402 Abb. H 26.

${ }^{61}$ Piranesi, Antichità Romane I, Taf. 24. 1. Gesamtkat. der Kupferstiche 2000 (note 59), p. 193, "Ninfeo di Nerone". 


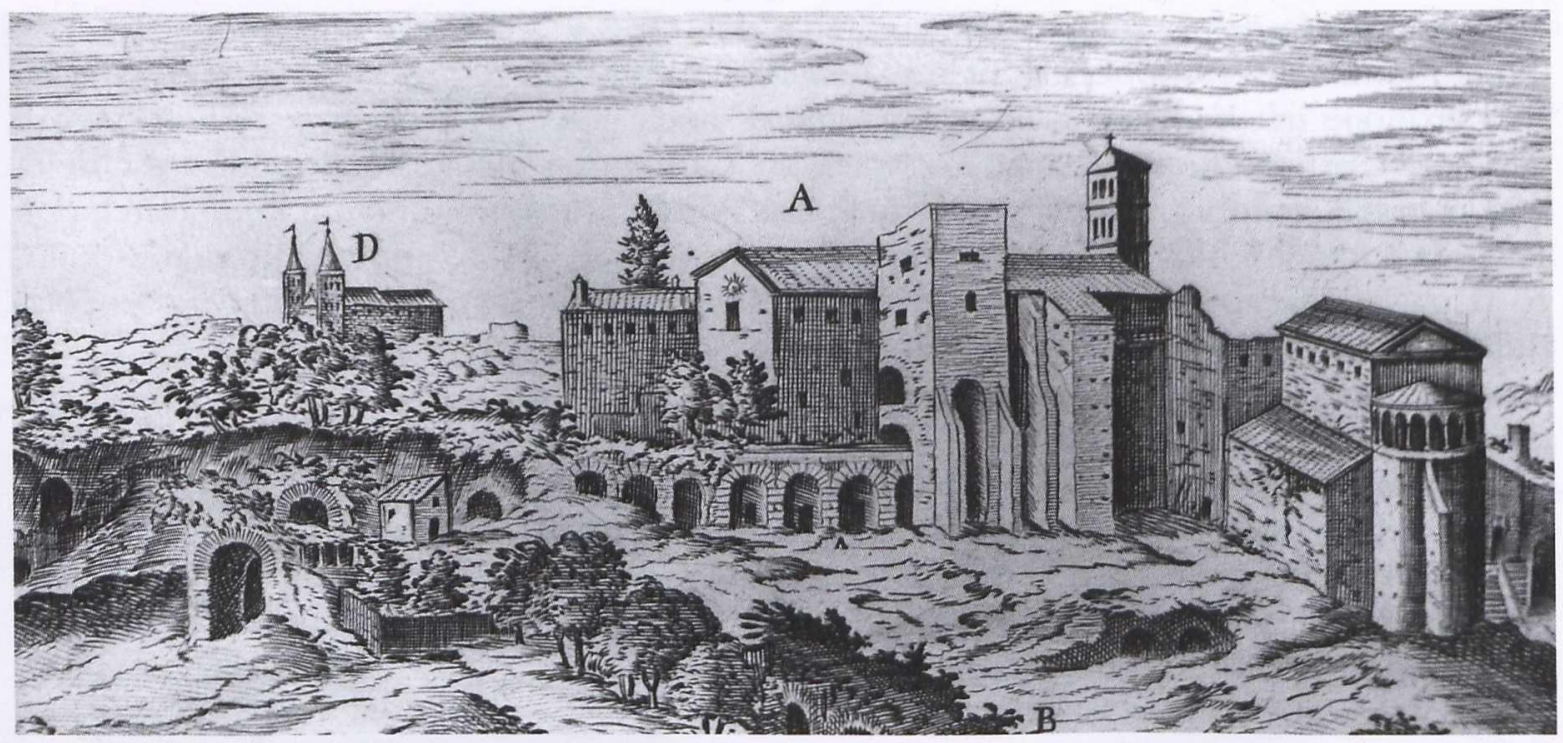

5 Die Curia Hostilia nach Dupérac (auf dem Podest des Claudiustempels). S. Dupérac, Vestigi di Antichità, 1575, pl. 14 (Ed. Losi, Roma, 1773). Universiteit Utrecht.

Giorgio Martini hat noch Reste davon erkennen können; er stellt sie als eine doppelläufige Anlage dar. ${ }^{62}$

Auf dem Podium waren keine antiken Strukturen erhalten. Man konnte sich dort einen beliebigen Bau vorstellen. Was Biondo vorschwebte, ist offen. Jedenfalls muß er sich den Bau dem gewaltigen Podium entsprechend imposant vorgestellt haben. Vielleicht war seine Vorstellung von den Curiae veteres inspiriert, die er identifizierte. Aber es ist auch möglich, daß er an etwas ähnliches wie große mittelalterliche Rathäuser dachte oder an einen Zentralbau, ähnlich wie der phantastische Sitz des Senats auf dem Kapitol gezeichnet wurde. Zumindest eine Basilika konnte in seinen Augen auch die Gestalt eines Zentralbaus einnehmen. So identifizierte er die Basilika, die Augustus nach Sueton im Namen seiner Neffen Caius und Lucius stiftete, mit dem später sog. Tempio della Minerva Medica bei Termini. ${ }^{63}$

$\mathrm{Zu}$ den Curiae veteres gehörten nach Biondo die diversen großen Ruinen, die bis heute über die gesamte Region der Capocce bzw. nach damaliger Bezeichnung Carinae hinweg verstreut liegen. ${ }^{64}$ Die Situation ist ersichtlich auf dem Romplan des Alessandro Strozzi (1474), dessen Angaben auf Biondo basieren. ${ }^{65}$ Der Abhang des Esquilin beim Kolosseum war durch mächtige Stützmauern eingefaßt, von denen heute noch Reste zu sehen sind; Rompläne der Renaissance zeigen noch mehr von ihnen. Auf dem Abhang ragen bis heute unter verstreuten Resten vier gewaltige, reich gegliederte Exedren auf. Sie gehörten nach späterer Erkenntnis zu den Trajansthermen, deren Disposition im wesentlichen den Diokletiansthermen glich. Aber für einen normalen Betrachter ist ein geordneter Zusammenhang schwer erkennbar. Biondo nahm die Exedren wohl für Teile von Hallen, die er für die Curiae veteres hielt.

${ }^{62}$ Uffizien, Gab. dei Disegni, A 327. O. Vasori, I monumenti antichi in Itallia nei disegni degli Uffizi, Roma, 1981, p.16.

63 Biondo, Roma inst. II 24; Sueton, Aug. 29.

${ }^{64}$ Biondo, Roma inst. II, p. 32.
${ }^{65}$ P. A. Frutaz, Le piante di Roma, Rom, 1962, Plan 89; G. Scaglia, 'The origin of an archeological plan of Rome by Alessandro Strozzi', Journal of the Warburg and Courtauld Institutes 27, 1964, pp. 137-163. 
Mit seiner Lokalisierung der Kurien auf den beiden Hügeln zu Seiten des Kolosseums, kreierte Biondo ein imposantes Regierungsviertel, das der Weltherrschaft der "Siegerin Rom" wahrhaft würdig war. Und das war offenbar auch seine wesentliche Absicht. Aus der Roma triumphans spricht seine Begeisterung für die Großartigkeit des antiken Rom. Das deutet schon der Titel des Werkes an. Besondere Hochachtung bringt Biondo der Regierung des Römischen Reichs entgegen. Er spricht sie als "geradezu göttliche Regierung eines Volkes an, das mächtiger und weiser als alle anderen war" ${ }^{66}$ Biondo stand mit seiner Bewunderung für die Größe des antiken Rom natürlich nicht allein. Sie steht wohl auch hinter Poggios Lokalisierung der Kurie auf dem Kapitol. Bei ihr muß man sich fragen: Wenn geistig so rege Humanisten wie Poggio wußten, wo sich das Senatulum befand, und verstanden, daß die Curia Hostilia nahe dabei und am Forum lag, und zudem bemerkten, daß der Bau von S. Adriano antik ist, wieso kamen sie dann nicht bereits auf den Gedanken, S. Adriano mit der Curia Hostilia zu identifizieren? Dafür kann ich nur eine Antwort sehen: Der Bau von S. Adriano erschien ihnen zu klein und zu unscheinbar für eine Kurie oder, anderes ausgedrückt, sie stellten sich die Kurie viel großartiger vor (vgl. fig. 3).

Aber Biondos Ausgangshypothese, daß die Erscheinung der Sitze der obersten Regierungsgremien im antiken Rom wie zu seiner eigenen Zeit der Würde der Gremien und der Macht des Staates entsprach, stimmt anscheinend nicht mit der Realität überein. Der Bau am Forum Romanum unterhalb des Kapitols, der heute als Curia Hostilia angesehen wird, ist nur klein und bescheiden gestaltet, obwohl er die Hauptkurie war und mehrfach in der großen Zeit des Römischen Imperium erneuert wurde, so klein, daß man sich fragt, wie der ganze Senat hineinpaßte. Sogar die altgriechischen Rathäuser waren aufwendiger. Der Bau umfaßt wirklich nur einen Saal, wie Alberti die Kurie beschreibt, ist aber noch bescheidener dekoriert als Alberti will. Vielleicht wollten die Römer ähnlich wie beim Tempel des Kapitolinischen Jupiter, dessen urtümliche etruskische Gestalt sie trotz vieler Erneuerungen bewahrten, auch bei der Kurie die Erinnerung an ihre primitive, aber heroische Frühzeit wach halten.

\section{Leon Battista Alberti}

Die einzige antike Schriftquelle über das Aussehen einer Kurie bildet Vitruvs Architekturtraktat. Ungeachtet der nachdrücklichen Ermahnung, die oben zitiert wurde, daß eine Kurie der Bedeutung des Staates entsprechen soll, widmet Vitruv ihr nur wenige karge Sätze, und mehr ist auch nicht nötig, denn die Beschreibung läuft auf einen so simplen Bau hinaus, wie es die Kurie am Forum ist. Anschließend an die Ermahnung heißt es wörtlich:

"Wenn sie quadratische Form hat, soll ihre Höhe mit 11⁄2 Breite festgelegt werden; wenn sie aber länglich ist, soll man Höhe und Breite zusammenzählen, und die Hälfte der Gesamtsumme soll man der Höhe bis unter die Decke zuweisen. Außerdem sind die Wände innen ringsum in halber Höhe mit Gesimsen aus Holzarbeit oder Stuck auszustatten". Das ist alles.

Biondo kannte diesen Text nicht. Trotz seiner umfassenden Kenntnis der antiken Literatur zitiert er nie Vitruv, und es läßt sich zeigen, daß er ihn merkwürdigerweise generell nicht berücksichtigt hat. ${ }^{67}$ Alberti hingegen nahm Vitruv als Basis für sein Architekturtraktat. Wie Vitruv stellt er Beschreibungen von Bautypen und Bauteilen in

${ }^{66}$ F. Biondo, Roma trionfante. Übers. L. Fauno, Venedig, 1544 , p. $92 \mathrm{v}$.
${ }^{67}$ H. Günther, 'Alberti, gli umanisti contemporanei e Vitruvio', in: Leon Battista Alberti. Architettura e cultura, Firenze, 1999, pp. 33-44. 
den Vordergrund. Nach dem Vorbild Vitruvs beschreibt er auch eine Kurie. ${ }^{6}$ Er beginnt mit einer kurzen historischen Einführung: Ursprünglich habe der Senat in Tempeln getagt, dann habe die Sitte zugenommen, die Sitzungen außerhalb der Stadt abzuhalten - das bezieht sich anscheinend auf die Curia Hostilia auf dem Caelius, weil der Hügel ursprünglich noch nicht zum Stadtgebiet Roms gehörte -, schließlich sei die Kurie mitten in die Stadt versetzt worden. Wohin sagt Alberti nicht. Man könnte denken, daß er das Forum Romanum als Standort annimmt. Aber eine solche Annahme läßt sich nicht verifizieren. Es ist ebenso denkbar, daß er die Kurie mit Poggio auf dem Kapitol lokalisierte. Seltsamerweise spricht Alberti nur von einer Kurie im Singular, geradezu als wüßte er nicht, daß es viele gab. Jedenfalls stimmt das nicht mit Biondo überein. Am auffälligsten wird der Unterschied zu Biondo beim Comitium. ${ }^{69}$ Alberti beschränkt sich auf die Mitteilung, daß in Rom ein eigener Platz für die Volksversammlungen bestimmt gewesen sei, ohne zu spezifizieren, wie er aussah oder wo er war. Kein Wort von Biondos großartiger Saepta auf dem Marsfeld.

Vitruv folgend, beschreibt Alberti dann die Kurie als einen Saal. Aber er bemüht sich, dessen Gestaltung entsprechend der Bedeutung einer Kurie aufzuwerten: Er behandelt den Gegenstand viel ausführlicher als Vitruv und gestaltet diesen Saal reicher und kunstvoller. Zunächst unterscheidet er nach Varro zwischen Priesterkurie und Senatskurie. Er hält sich wie gewöhnlich so auch bei den Kurien wieder an konkrete Vorbilder, nur identifiziert er sie wie üblich nicht ausdrücklich. ${ }^{70}$ Wer Rom gut kannte, konnte trotzdem oft erkennen, worauf sich Alberti bezieht.

Bei beiden Arten von Kurien berücksichtigt Aberti nicht die äußere Erscheinung, sondern nur den Innenraum. Die Priesterkurie soll wie ein Tempel gestaltet sein: als rechteckiger Saal mit Eingang an einer Schmalseite und Exedra gegenüber, tonnengewölbt, ohne Fenster (fig. 6a, b); zudem führt Alberti ein kunstvolle Gliederung ein, unten mit Säulen vor den Wänden, darüber eine niedrige Zone mit Statuen in Nischen. Die Priesterkurie gleicht demnach in ihrer Disposition, Gliederung und sogar in den Proportionen dem Tempel der Venus und Roma. Die Übereinstimmung ist aus mehreren Gründen sinnvoll. Erstens tagte der Senat - wie Poggio bemerkt ${ }^{71}$ - manchmal im Tempel der Venus und Roma. Zweitens sind in diesem Bau zwei gleiche Tempel miteinander verbunden, und das paßt zum Gebrauch des Plurals für die Bezeichnung der Curiae veteres. Daß der Tempel allseits von einer Peristase umgeben war, wußte man nicht in der Renaissance.

Die Senatskurie besteht auch nur aus einem Saal, aber wie es zu einem gewöhnlichen profanen Raum paßt, ist dieser Saal breiter proportioniert als die Priesterkurie oder ein Tempel und hat keine Apsis, er ist nur mit einer flachen Holzdecke gedeckt und hat einen hohen Obergaden mit Fenstern (fig. 7a, b). Alberti begründet diese Eigenheiten damit, daß gute Beleuchtung nötig sei, weil in der Senatskurie viel geschrieben werde, und daß Flachdecken besser für die Akustik als Gewölbe und daher günstiger für die Reden seien, die in der Senatskurie gehalten würden. Nach Albertis Beschreibung gleicht die Senatskurie in Disposition, Gliederung und Proportionen zwei Hallen, die auf dem

\footnotetext{
68 Auf die Ankündigung in De re aed. VIII 8, daß im folgenden Komitien, Kurie und Senat behandelt würden, folgt in VIII 9. Ed. Theuer, 460, die wiedergebene Passage.

${ }^{69}$ De re aed. VIII 9. Theuer, p. $459 \mathrm{ff}$.

${ }^{70}$ Vgl. zu Problem und Methode Alberti, De re aed. VI 1.
}

${ }^{71}$ De varietate fortunae, Valentini/ Zucchetti IV (note 18), p. 234. Den in der Historia Augusta, Valerian, XXII.5.4, u.a. Schriften gen. Dioskurentempel identifiziert Poggio, wie es seinerzeit mehrfach geschah, mit der heute als Tempel der Venus und Roma angesehenen Ruine hinter S. Maria Nuova. 


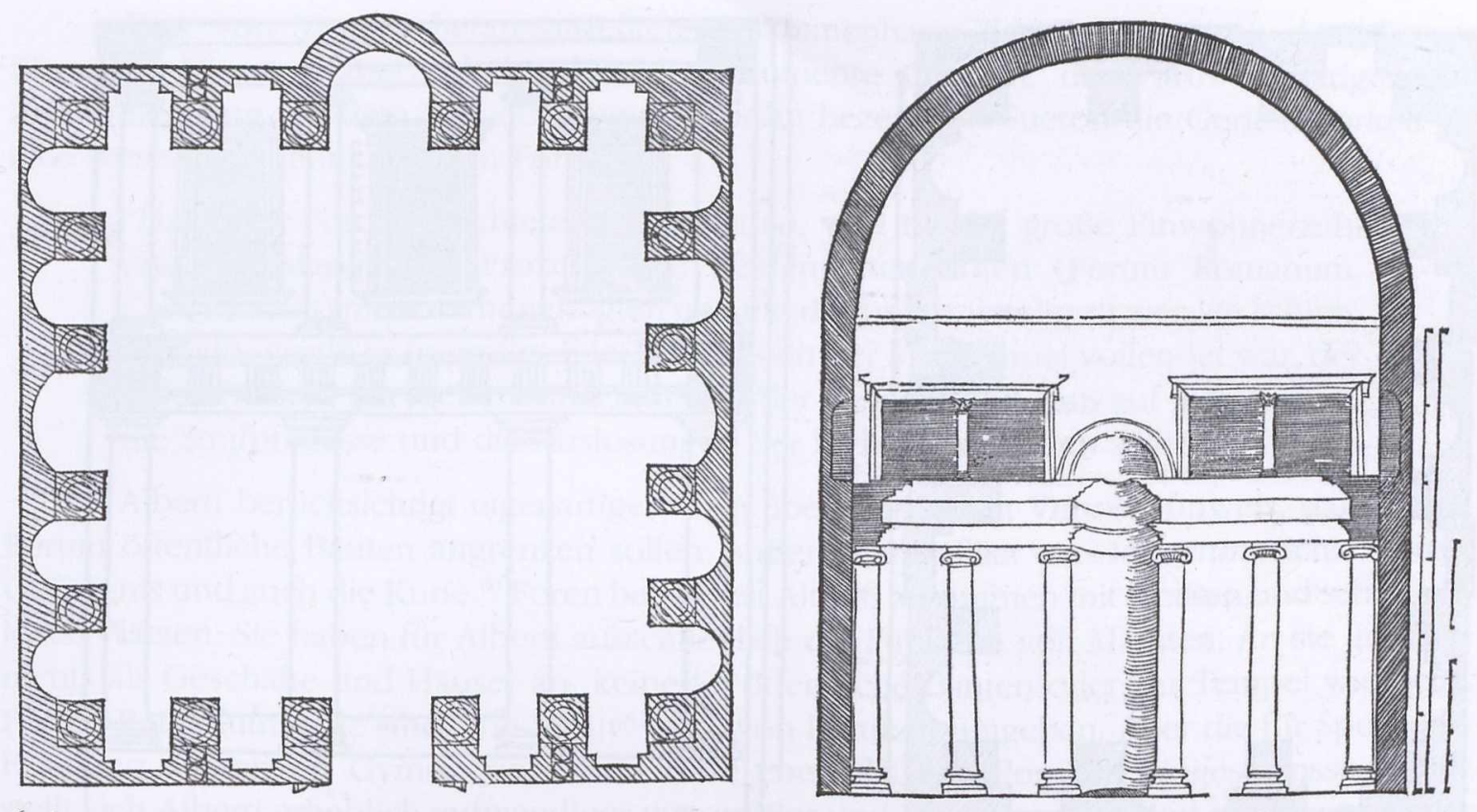

6a,b Die Priesterkurie nach Alberti, De re aedificatoria, L.VIII c.9 (Ed. Bartoli, Firenze, 1550). Universiteit Utrecht.

Palatin standen, so wie sie vom 18. Jahrhundert bis heute rekonstruiert warden. ${ }^{72}$ Jetzt sind nur noch wenige Reste von den Hallen erhalten; sie gelten als Teile der Domus Flavia.

Aber Alberti bleibt nicht bei dem einen Saal. An anderer Stelle, im Zusammenhang mit der Behandlung der Funktionen, liefert er weitere Angaben zur Senatskurie. ${ }^{73}$ Der Eingang in den Senat (in senatum aditus) soll mehr gesichert (munitus) als übermäßig würdig (bonestus) sein, weil immer wieder die Gefahr eines Volksaufstandes drohe. Das ist eine freie Erfindung, vielleicht erwachsen aus dem Andenken an die alte Burg auf dem Kapitol. Vor allem stellt sich Alberti vor, daß die Senatskurie mit einem Ensemble von Bauten verbunden sei. Dazu gehören ein Tempel und eine Curia iudiciaria, das soll wohl eine Basilika sein. Alberti kommentiert, diese Verbindung sei nützlich, weil die Senatoren in der Curia iudiciaria ihren anderen Geschäfte nachgehen könnten und weil sie fromm seien. "Dazu kommt, daß es ja für den Staat nötig ist, wenn der Senat Gesandte oder Fürsten fremder Völker empfangen soll, einen Ort zu haben, wo man die Gäste so aufnehmen kann, wie es der Würde von ihnen und der Stadt entspricht". Daß bei der Kurie Gesandte fremder Völker empfangen wurden, belegt der schon mehrfach zitierte grundlegende Passus des Varro über die Kurien: Demnach gehört zur Kurie ein Platz, genannt Graecostasis, wo die Gesandten warteten. ${ }^{74}$ Alberti mag durch

\footnotetext{
${ }^{72}$ F. Bianchini, Il palazzo dei Cesari, Verona, 1738; J. Durm, Die Baustile II 2. Die Baukunst der Etrusker. Die Baukunst der Römer (Handbuch der Architektur), Stuttgart, 1905 (1. Ed. 1884), p. 516 Fig. 578; S. Gibson, J. Delaine, A. Claridge, 'The triclinium of the Domus Flavia: a new reconstruction', Papers of the British School at Rome 62, 1994, pp. 67-97; M. Royo,
}

Domus Imperatoriae. Topographie, formation et imaginaire des palais impériaux du Palatin, 1999, pp. 303-368; P. Gros, L'Architecture romaine du début du III siècle av.J.-C. à la fin du Haut-Empire, Paris, 2002, Bd. II, p. 252ff.

${ }^{73}$ De re aed. V 9. Theuer, p. 244.

74 Varro, Ling. lat. V 155. 

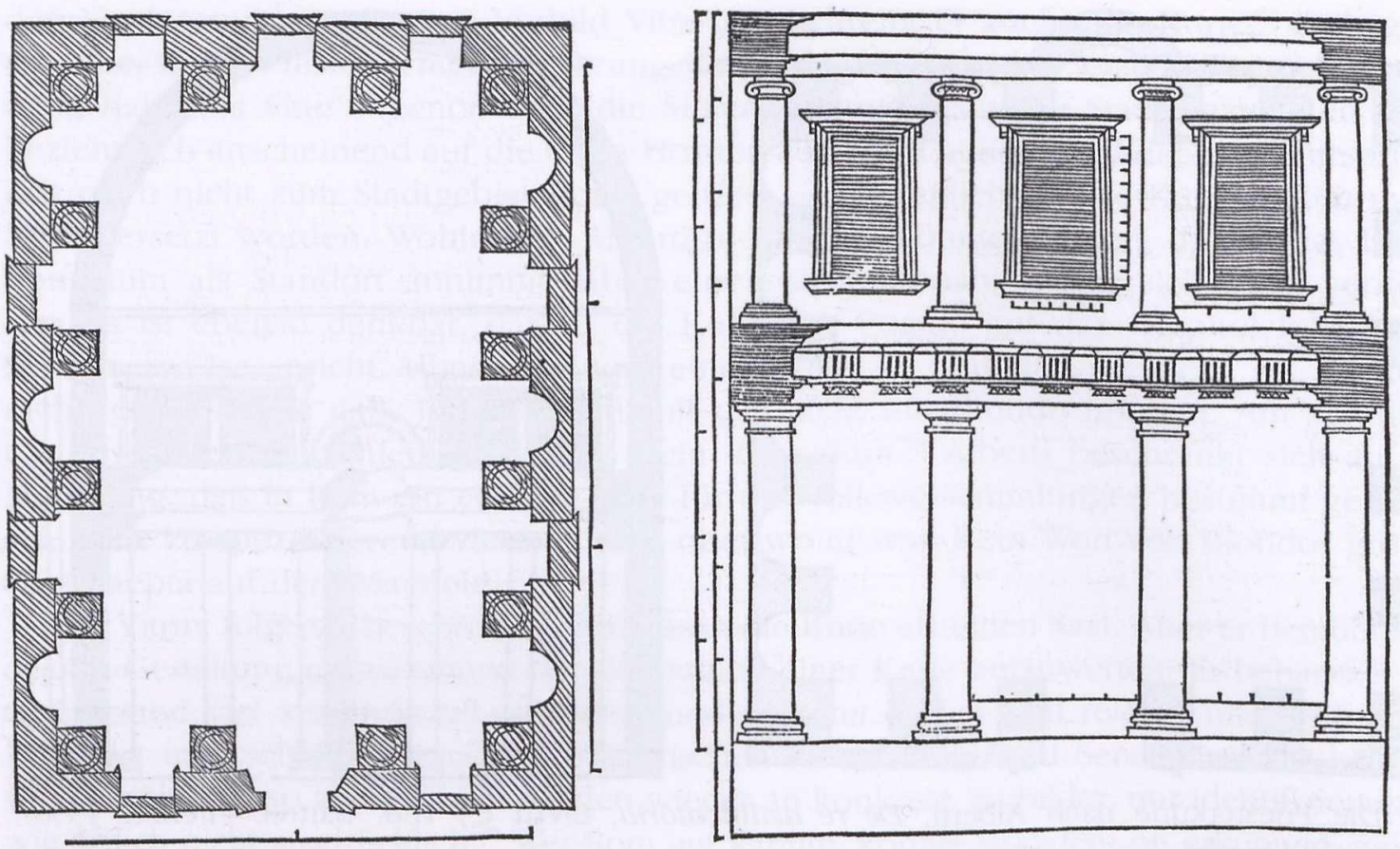

7a,b Die Senatskurie nach Alberti, De re aedificatoria L. VIII, c. 9 (Ed. Bartoli, Firenze, 1550). Universiteit Utrecht.

das Forum Romanum dazu angeregt worden sein, eigenmächtig die Verbindung der Kurie mit Tempel und Basilika einzuführen. Zudem gibt Vitruv an, die Basilika, die er in Fanum errichtete, sei mit einem Tempel verbunden gewesen. ${ }^{75}$ Aber vor allem bemerkte Alberti offenbar, daß die Kurie auch so, wie er sie ausschmückt, immer noch viel zu unscheinbar ist, um die Größe des Romischen Reichs zu repräsentieren. Deshalb müssen Tempel und Basilika hinzukommen. Sie behandelt Alberti als besondere Höhepunkte der Architektur; den Tempel hält er mit Vitruv für die vornehmste von allen Bauaufgaben. ${ }^{76}$

Die Basiliken behandelt Alberti zusammen mit den Tempeln im Buch über die Sakralbauten. Selbstverständlich wußte er aus Vitruv, daß sie für die Rechtsprechung dienten, und leitet davon auch ihre Disposition ab. Aber die profane Funktion stellt er nur als ihre ursprüngliche Bestimmung hin: "Zweifellos waren die Basiliken anfangs jener Ort, wo die Staatsmänner unter einem Dach zusammenkamen, um Recht zu sprechen". ${ }^{77}$ Im übrigen gleicht Alberti die Basiliken Sakralbauten an. Er hält sich ganz an die konstantinischen oder andere frühchristliche Basiliken in Rom. Es war damals und auch später in der Renaissance durchaus nicht üblich, sich antike Basiliken generell wie die frühen christlichen Kirchen vorzustellen. Alberti beschreibt nach diesem Vorbild nicht nur die Disposition, sondern auch den Schmuck der Basiliken. Er entfernt sich dabei beträchtlich von Vitruvs Beschreibung der Basilika in Fanum. Das war ein Holzbau; von Schmuck ist dort überhaupt keine Rede. Die enorme Pracht, mit der Alberti Basiliken ausgestattet haben will, paßt nur zu Sakralbauten.

75 Vitruv V 1 (7).

77 De re aed. IX 14. Theuer, p. 393

${ }^{76}$ De re aed. VII. Theuer, p. 349. 
Den von Vitruv ${ }^{78}$ herausgestellten Zusammenhang der Basiliken mit dem Forum übergeht Alberti. Dabei gab es diverse prominente Quellen, die Vitruv bestätigen. Im Zusammenhang mit dem Bau des Augustusforum bezeichnet Sueton die Gerichtsbarkeit als eine wesentliche Funktion von Foren:

"Das neue Forum errichtete er (Augustus), weil für die große Einwohnerzahl und die Menge der Prozesse die beiden alten Foren (Forum Romanum, Caesarforum) nicht mehr genügten und ein drittes notwendig zu werden schien; deshalb wurde es in großer Eile, bevor noch der Marstempel vollendet war, der Öffentlichkeit übergeben, und zwar mit der Bestimmung, daß auf ihm speziell die Strafprozesse und die Auslosungen der Richter stattfinden sollten". ${ }^{79}$

Alberti berücksichtigt eigenartigerweise überhaupt nicht Vitruvs Hinweis, daß an ein Forum öffentliche Bauten angrenzen sollen, außer der Basilika ein Aerarium (Schatzhaus), Gefängnis und auch die Kurie. ${ }^{80}$ Foren behandelt Alberti zusammen mit Straßen und schmucklosen Plätzen. Sie haben für Alberti ausschließlich die Funktion von Märkten. An sie grenzen nichts als Geschäfte und Häuser an, keinerlei öffentliche Bauten oder gar Tempel wie beim Forum Romanum. ${ }^{81}$ Sie sind, wie Vitruv ${ }^{82}$ will, von Portiken umgeben. Aber die für Sport und Erholung dienenden Gymnasien, deren Höfe ebenfalls von Portiken eingeschlossen sind, stellt sich Alberti erheblich aufwendiger vor, größer und prächtiger: sie sind mit Marmor ausgekleidet und mit Mosaik gepflastert. Derartigen Schmuck sieht Alberti für das Forum nicht vor. Beim Forum beschreibt er die Portiken längst nicht so ausführlich wie beim Gymnasium. Alberti sagt sogar ausdrücklich, daß die Säulenhallen der Gymnasien geräumiger als diejenigen der Foren sein sollen. ${ }^{83}$

\section{Lokalisierung der Kurien im 16. Jahrhundert}

Im Lauf der Renaissance setzte sich bei Humanisten die Erkenntnis durch, daß das Kapitol nicht der Regierungssitz des antiken Rom war und daß auf dem Hügel hauptsächlich Tempel und Heiligtümer standen. Die antiken Schriften berichten von einigen Dutzenden von ihnen, so daß sich Michel de Montaigne wunderte, wie so viele Bauten überhaupt Platz auf dem ziemlich kleinen Hügel gefunden haben könnten. ${ }^{84}$

Biondos Lokalisierung der Kurien hatte teilweise bis ins 19. Jahrhundert Bestand, auch wenn sie vielfach auf Widerspruch stieß. Aber seine Vorstellung, daß die Curiae veteres so monumental waren, wie es der Herrlichkeit des Römischen Reichs entspricht, verlor trotzdem an Gewicht. Schon im 16. Jahrhundert hatte sich die Erkenntnis durchgesetzt, daß die großen Ruinen auf dem Esquilin zu den Trajansthermen und Titusthermen gehörten. Die Curiae veteres wurden zwar weiterhin auf dem Esquilin lokalisiert, aber jetzt nahe bei S. Pietro in Vincoli. Ausschlaggebend dafür war nur noch die Deutung der Ortsangaben in den Schriften, nicht mehr die Attraktion markanter Ruinen. So verband sich mit ihrer Lokalisierung keine konkrete Vorstellung von ihrem Aussehen. Der gelehrte Vitruv-Übersetzer Fabio Calvo markiert in seinem Plan der Roma quadrata, des ältesten Rom, eine Senatskurie inmitten der Stadt, eine Domus Sacerdotum auf dem Palatin und eine Curia vetus auf dem Esquilin (fig. 8). ${ }^{85}$ Die Curia

\footnotetext{
78 Vitruv V 1.

79 Sueton, Aug. 29.

80 Vitruv V 2.

${ }^{81}$ De re aed. VIII 6. vgl. V 18. VII 1. Theuer, pp. 437ff, $284,346$.
}

\footnotetext{
82 Vitruv V 1.

${ }^{83}$ De re aed. VIII 8. Theuer, p. 457.

${ }^{84} \mathrm{M}$. de Montaigne, Journal de voyage en Italie, Ed. P. Michel, Paris, 1974 , p. $256 \mathrm{ff}$.

${ }^{85}$ Frutaz 1962 (note 65), Plan 7.
} 


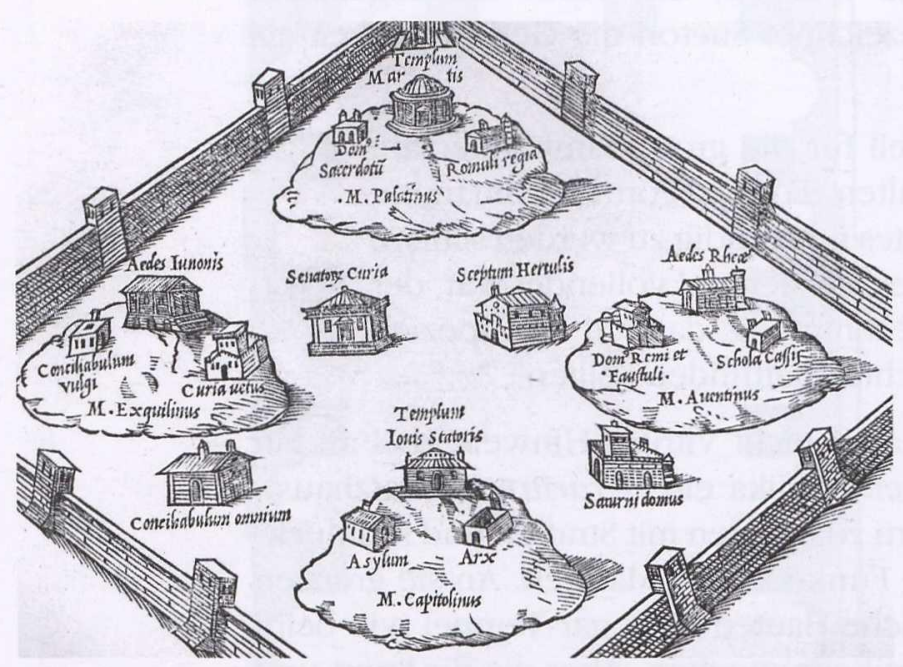

8 Roma quadrata: die Stadt in der Zeit von Romulus, mit der Kurie des Senats im Stadtzen-trum und der Curia vetus auf dem Esquilin. Stadtplan des Fabio Calvo (Frutaz 1962, pl. 7).

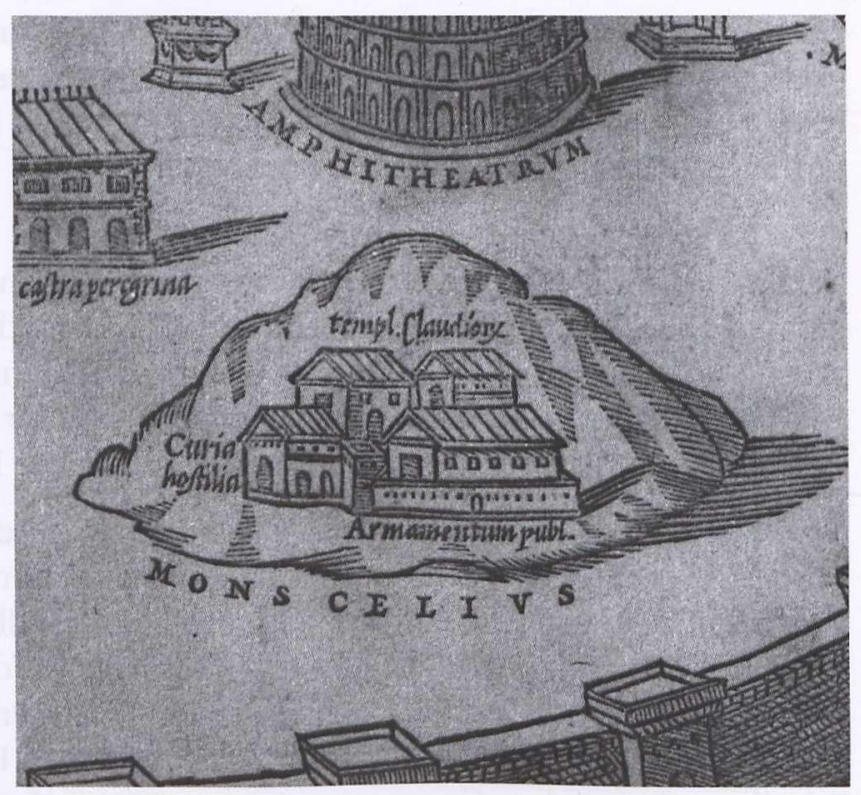

9 Die Curia Hostilia auf dem Mons Caelius. Ausschnitt aus dem Stadplan von Rom zur Zeit der Flavier von Fabio Calvo, 1527 (Frutaz 1962, pl. 10).

vetus sieht wie ein mittelalterliches Kastell aus. Diese Darstellung war vielleicht durch die befestigte Anlage des Komplexes von S. Pietro in Vincoli angeregt. Eine spätere Abbildung der Curiae veteres ist mir nicht bekannt.

Die Lokalisierung der Curia Hostilia auf dem Caelius fand im Allgemeinen Zustimmung. Bald wurde auch Albertis Idee übernommen, die Kurie als Teil einer Gruppe von Bauten zu rekonstruieren, zuerst wohl von Fabio Calvo in seinem Plan Roms zur Zeit der Flavier (1527) (fig. 9). ${ }^{86}$ Calvo stellt über den Substruktionen des Claudius-Tempels auf dem Caelius ein Ensemble von vier eng zusammenstehenden Bauten und einem Turm dar, der vermutlich den Nordeingang bewacht. Die Bauten sind bezeichnet als Curia Hostilia (links), Templum Claudiorum (oben) und Armamentum publicum. Calvo richtet sich nach dem von Pomponius Laetus interpolierten antiken Regionenkatalog von Rom. Dort sind auf dem Caelius aufgelistet die Residenz (Regia) des Tullus Hostilius und der Tempel, den Tullus Hostilius in eine Kurie umwandeln ließ, ${ }^{87}$ sowie der Tempel des Claudius und das Arsenal (Armamentum). ${ }^{8}$ Aus der Liste allein erklärt sich das Ensemble aber nicht. Calvo hat seine vier Bauten aus insgesamt zwanzig ausgewählt, die dort aufgeführt sind, und er hat sie hier ausnahmsweise als zusammenhängendes Ensemble dargestellt im Unterschied zu den übrigen Regionen, wo die Bauten separat dargestellt sind.

Die Verbindung der Kurie mit einem Tempel, und ebenso der wehrhafte Eingang orientieren sich wohl an Alberti. Die Einbeziehung des Arsenals ist wohl durch die Tradition mittelalterlicher Rathäuser angeregt. In der Halle beim alten Eingang in den Palazzo

86 Frutaz 1962 (note 65), Plan 10; P. N. Pagliara, 'La Roma antica di Fabio Calvo. Note sulla cultura antiquaria e architettonica', Psicon. Rivista internaz. di Architettura 2, 1976, Nr. 8-9, pp. 65-87.
87 Von Pomponius nach Livius ergänzt. Livius I 30; 33, 2.

88 Valentini/ Zucchetti I (note 18), p. $209 f$ f. 
Vecchio in Florenz war eine Waffenkammer. Francesco di Giorgio schließt in ein ideales Rathaus ein armamentario ein, wo man die gesamte Artillerie der Gemeinde aufbewahren soll. ${ }^{89}$ Pietro Cataneo will, daß das Zeughaus, in dem die Artillerie deponiert ist, direkt beim Rathaus liegt. ${ }^{00}$ Die Berichte über das Armamentum, das der Architekt Philon von Eleusis für die Athener in Piraeus errichtete (zw. 347 u. 330 v.Chr.), sprachen dafür, sich antike Zeughäuser großartig vorzustellen. Es war wegen seiner Größe und seiner Schönheit hochberühmt in der Antike, und das war von Anfang der Renaissance an bekannt. ${ }^{91}$

\section{Daniele Barbaro und Andrea Palladio}

Daniele Barbaro und Palladio machten wesentliche Fortschritte im Verständnis der Antike. Palladios Lehre von den Säulenordnungen zeugt von einem neuen Blick auf die Ruinen, weil endlich das Pathos der Tempelportiken mit ihren engen Interkolumnien wahrgenommen und als essentielles architektonisches Motiv gewertet wird. ${ }^{92}$ Wie ideenreich sich Barbaro und Palladio theoretisch mit der Antike auseinandersetzten, zeigen ihre Abhandlungen über antike Staatsbauten. Sie fanden auf der Basis von Vitruv einen originären Weg, um ein großartiges Regierungszentrum zu
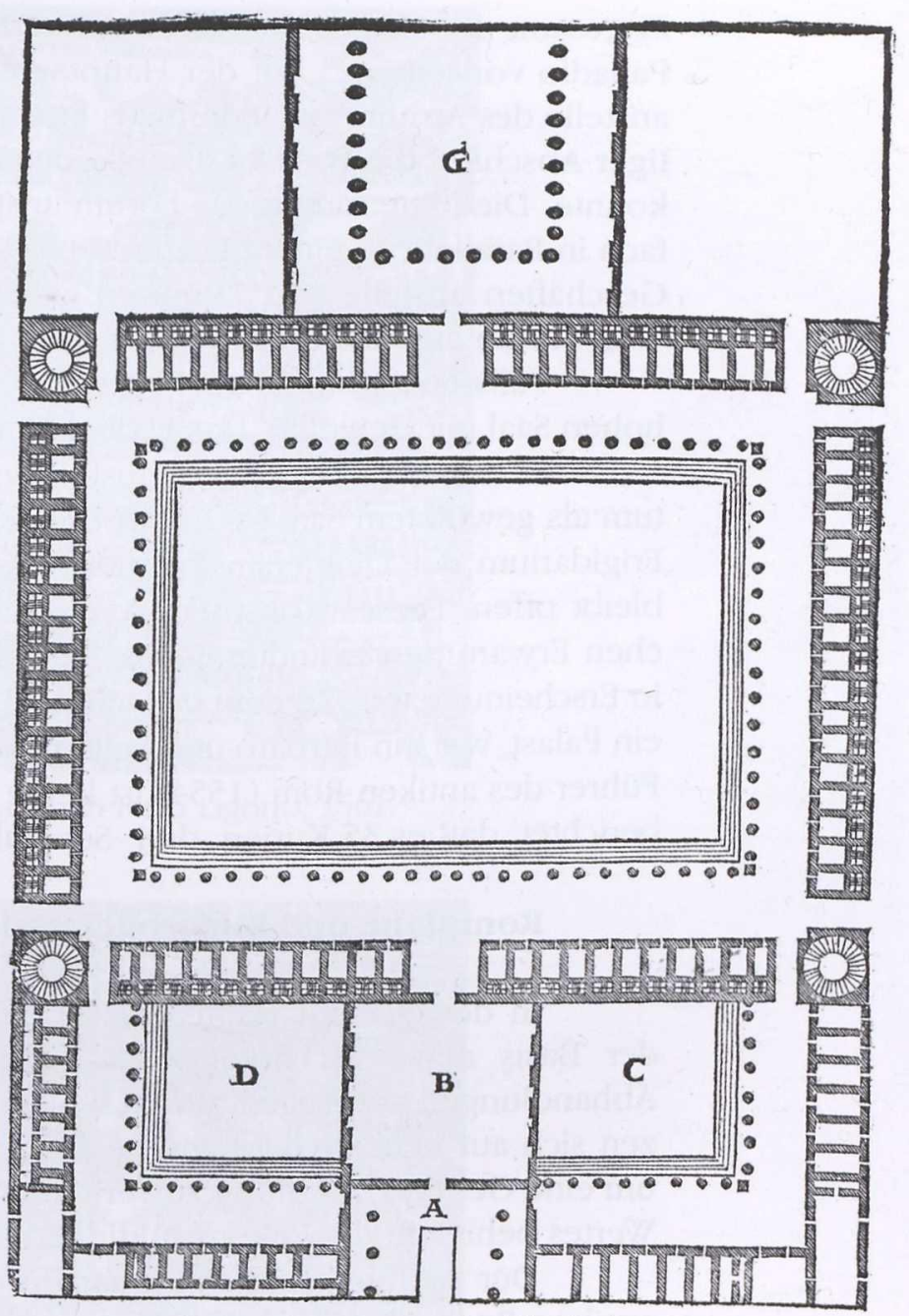

10 Rekonstruktion der Kurie als Theil des Forums, nach Andrea Palladio und Daniele Barbaro, Vitruvedition 1567; $B=$ Curia. Universiteit Utrecht. imaginieren.93 Ihr Ausgangspunkt ist gerade Vitruvs Angabe, daß ans Forum Staatsbauten wie Basilika, Kurie und andere angrenzen sollen. Auch sie kommen nicht daran vorbei, daß Vitruv nur einen Saal für die Kurie beschreibt. Aber dieser Saal wird zum Bestandteil eines großen geschlossenen "Palazzo", wie Barbaro ausdrücklich formuliert (fig. 10). In der Mitte dieses Palastes liegt das Forum als von Portiken eingegrenzter Hof, daran grenzen an Basilika, Kurie, Sekretariate für den Senat, Gefängnis und Münze (zecca). Albertis Angabe folgend, daß die Gestaltung von Privathäusern öffentliche Bauten nachahmt, ist die Anlage

${ }^{89}$ F. di Giorgio, Trattati 1967 (note 29), 350, Taf. 206. Auch in Deutschland dienten damals die Rathäuser als Zeughaus. Albrecht 2004 (note 3), p. 13.

90 P. Cataneo, I quattro primi libri di architettura, Venezia, 1554, p. 10v.

91 Valerius Maximus, Factorum ac dictorum memorabilium VIII 12.2; Plinius, Nat.hist. VII 125; Vitruv, VII Praef.
92 H. Günther, 'Palladio e gli ordini di colonne', in: Andrea Palladio. Nuovi Contributi, Milano, 1990 , pp. 182-197.

${ }^{3}$ Vitruv Ed. D. Barbaro 1556 u. 1567 (Reprint Milano 1567 mit Kommentaren von M. Tafuri u. M. Morresi). Ed. 1567 , pp. 207-210 mit Illustration des Grundrisses eines Forum. Palladio 1570 , III c. $16-18$; p. 36 Grundriß eines Forum. 
insgesamt der Disposition eines antiken Hauses angeglichen, so wie sie sich Barbaro und Palladio vorstellten. ${ }^{94}$ Auf der Hauptachse liegen vorn ein Vestibül, anschließend die Kurie anstelle des Atrium/Cavaedium als Prachtraum und Empfangshalle des Hauses, als rückwärtiger Abschluß die Basilika anstelle des Tabularium, das die Form einer Basilika annehmen konnte. Die Mitte nimmt das Forum anstelle des Peristyl des Hauses ein, den Alberti mehrfach in Parallele zu einem Forum setzt.95 Das Forum ist, wie Alberti will, ganz umgeben von Geschäften anstelle von Zimmern, aber dahinter liegen Kurie und Basilika, die ebenfalls vom Forum aus direkt zugänglich sind.

Palladio illustriert auch den Querschnitt dieses Palastes: Die Kurie bildet da einen hohen Saal mit Gewölbe. Das widerspricht Albertis Rat, daß die Senatskurie flachgedeckt sein soll, aber es entspricht Albertis ausführlicher Beschreibung des Atrium/Cavaedium testudinatum als gewölbtem Saal in der Art des vermeintlichen Atrium bzw., wie man heute annimmt, Frigidarium der Diokletiansthermen. Wie der Regierungspalast von außen aussehen sollte, bleibt offen. Dieses Konstrukt demonstriert, wie sich Vitruvs Angaben mit der herkömmlichen Erwartung verbinden ließen, daß das Regierungszentrum auch in der Antike großartig in Erscheinung trat. Zu dem gewaltigen Podest des Claudius-Tempels auf dem Caelius würde ein Palast, wie ihn Barbaro und Palladio ausgedacht haben, gut passen. Allerdings in Palladios Führer des antiken Rom (1554) ist keine Rede von einem solchen Regierungspalast. Da wird berichtet, daß es 35 Kurien, drei Senatuli, das ,Sekretariat’ bei S. Martina etc. gab. ${ }^{96}$

\section{Rompläne und Bilderbücher des antiken Rom aus der Spätrenaissance}

In der zweiten Hälfte des 16. Jahrhunderts verbreiteten sich Publikationen, die auf der Basis neuer archäologischer Erkenntnisse anschaulicher als die früheren gelehrten Abhandlungen vor Augen führen wollten, wie Rom in der Antike als Ganzes aussah. Sie stützen sich auf neue archäologische Erkenntnisse, aber sie führen auch freie Erfindungen ein, um eine Gesamtvorstellung zu vermitteln. Ungeachtet ihres eingeschränkten archäologischen Wertes behielten die Rekonstruktionen lange Geltung.

Der Architekt und Antiquar Pirro Ligorio publizierte 1561 eine Rekonstruktion des antiken Rom, die eine lebendige Vorstellung von der alten Erscheinung der Stadt vermittelt. ${ }^{97}$ Die meisten bedeutenden Bauten sind erfaßt. Bei erhaltenen Bauten gibt Ligorio im Allgemeinen den Bestand gut wieder. Sorgfältig berücksichtigt er, wo verschwundene Bauten lokalisiert wurden und was die antiken Schriften über sie überliefern. Teilweise richtet sich die Darstellung der verschwundenen Bauten nach Münzen. Manchmal ist allerdings ganz eigenständig interpoliert. Ligorio hatte da Übung; er neigte auch sonst gelegentlich dazu, archäologische Fakten mit eigenen Erfindungen zu kombinieren, ohne offenzulegen, wie die Grenzen dazwischen liegen. Solche freien Erfindungen bilden die Darstellungen der Curia Hostilia auf dem Caelius oder der Kurie und des Senatulum im Zentrum, beim Forum

94 Vitruv 1567, 280. Palladio 1570, II c. 4-7, pp. 25, 34.

95 Alberti, De re aed. V 2; V 17. Theuer, pp. 224, 273 (Alberti bezeichnet den mittleren Hof als Atrium; vgl. dazu meine ob. zit. Artikel zu den Vorstellungen vom antiken Haus in der Renaissance).

96 A. Palladio, Le antichità di Roma, Roma, 1614, pp. 21-27.

97 Frutaz 1962 (note 65), Plan 17; H. Burns, 'Pirro Ligorio's reconstruction of ancient Rome: The antiquae urbis imago of 1561', in: R. W. Gaston (ed.), Pirro Ligorio. Artist and Antiquarian, Firenze,
1988, pp. 19-92; M. M. McGowan, 'Unwillkürliches Gedächtnis - Romerfahrung in der Spätrenaissance', in: Ruinenbilder, München, 2002, pp. 17-30; B. Palma Venetucci (ed.), Pirro Ligorio e le erme di Roma, Roma, 1998. Vgl. allg. zu Ligorio und seinen Bildungshorizont als Antiquar: A. Scheurs, Antikenbild und Kunstanschauungen des neapolitanischen Malers, Architekten und Antiquars Pirro Ligorio (1513-1583), Köln, 2000; D. R. Coffin, Pirro Ligorio. The Renaissance artist, architect and antiquarian, University Park, 2004. 


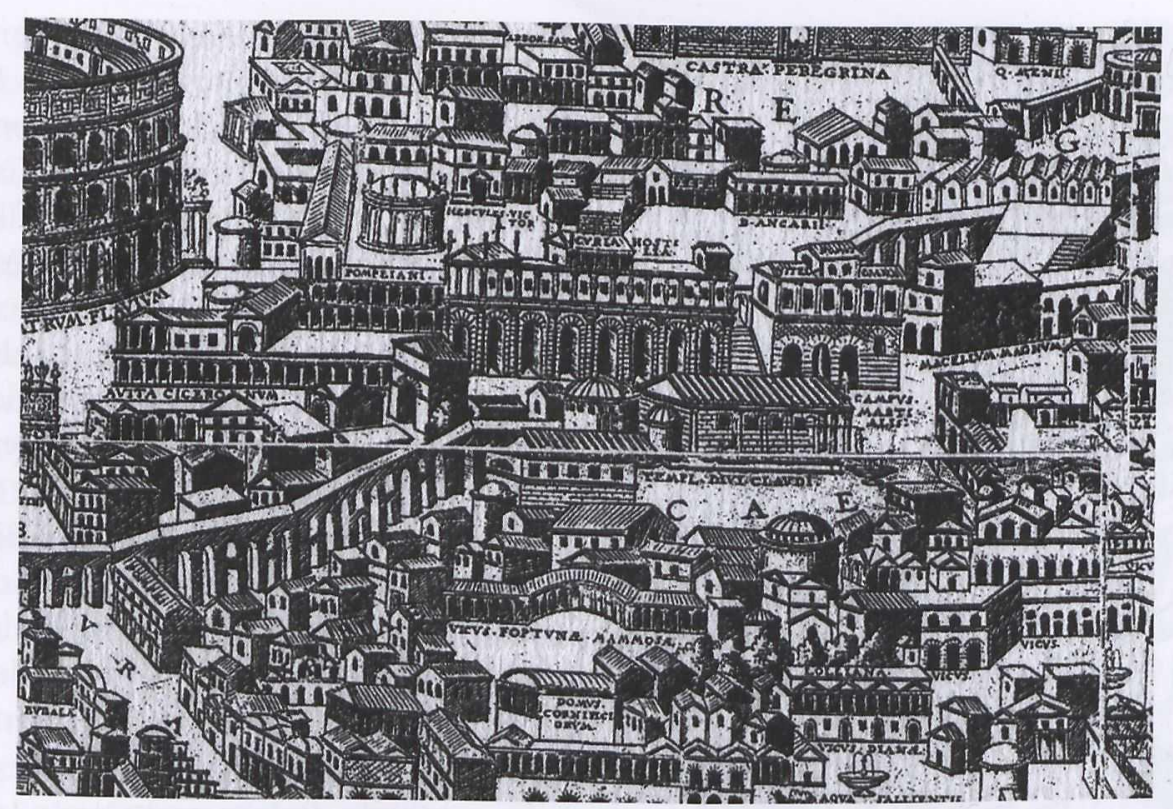

11 Die Curia Hostilia auf dem Mons Caelius, nach Pirro Ligorio, 1561.

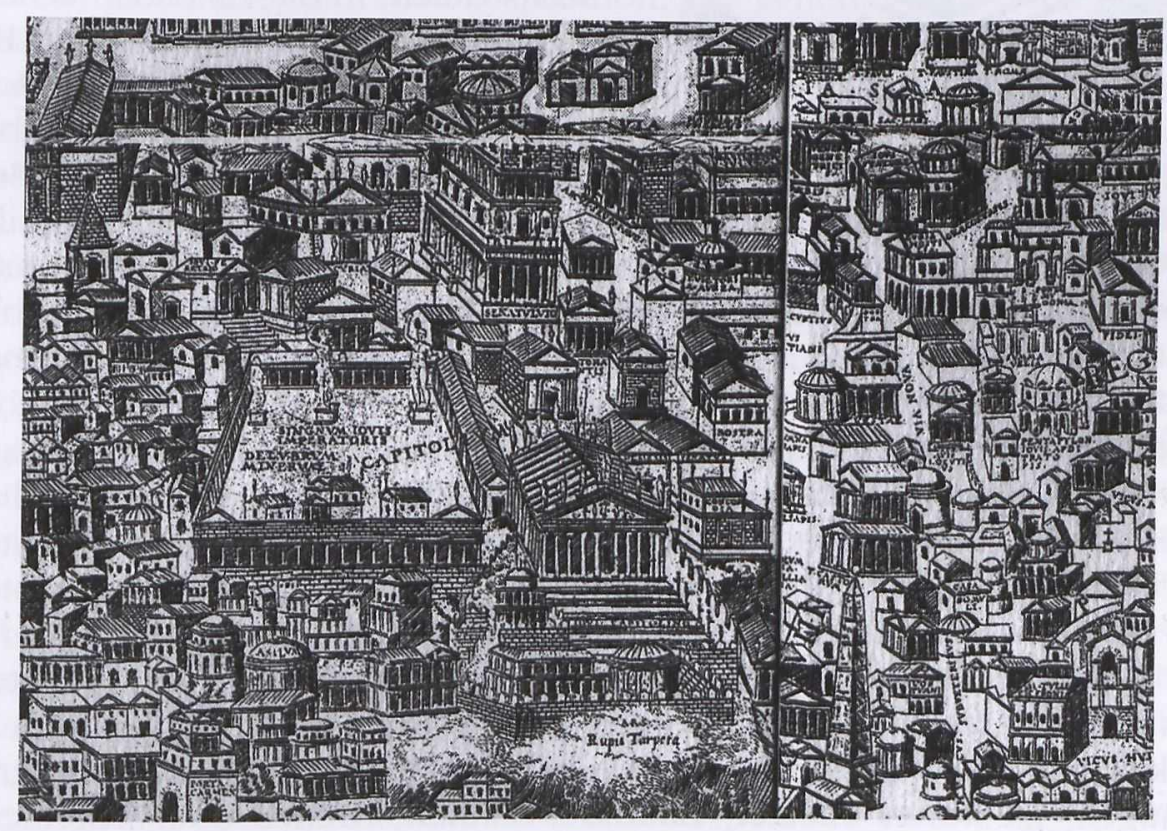

12 Kapitol und Forum Romanum nach Pirro Ligorio, 1561 (hinter dem Kapitol rechts Senatulum, links Kurie).

Romanum (fig. 11, 12). Bei der Gestaltung der Kurie im Zentrum war Ligorio frei, denn niemand kannte damals Relikte von diesem Bau. Beim Senatulum berücksichtigt er den antiken Bau von S. Martina nicht, sondern erfindet ein großartiges Hochhaus. In seiner Darstellung der Curia Hostilia verschmelzen die Arkaden des Podiums des Claudius-Tempels mit einem langgestreckten Obergeschoß und einem tempelartigen Bau darauf zu einer neuen Einheit. Ligorios Romplan fand rasch Nachfolge. Étienne Dupérac, der seit 1559 in Rom gelebt hatte, publizierte 1574 eine noch sorgfältiger ausgeschmückte, bedachtsam überarbeitete, 


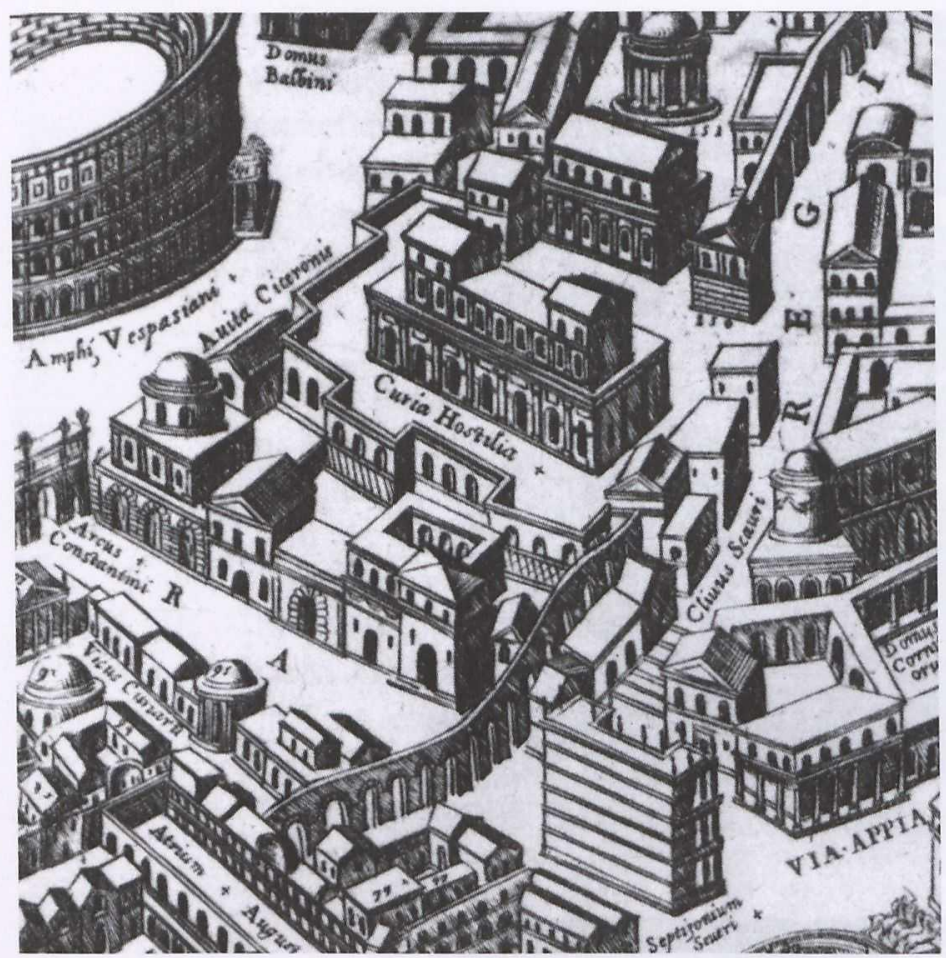

13 Die Curia Hostilia auf dem Mons Caelius, nach S. Dupérac, 1574.

aber nicht immer wirklich verbesserte Fassung von Ligorios Romplan (fig.13). ${ }^{98}$ Fünf Jahre später erschien ein ähnlicher Romplan von der Hand des Stechers Mario Cartaro, der hauptsächlich fremde Druckgrafik kopierte und in den Handel brachte. ${ }^{99}$ Er hält sich an Dupérac, korrigiert ihn teilweise seinerseits, aber weniger mit Bedacht, als mit freier Phantasie. Ligorios Rekonstruktionen sind noch bei F. H. Köhler, Urbs Roma, Leipzig 1829, paraphrasiert.

Inzwischen erschienen Bilderbücher über das antike Rom im Druck. Zunächst enthielten sie nur Abbildungen von realen Bauten, wie Serlios drittes Buch (1540) oder Palladios viertes Buch (1570). Dann kam eine neue Art auf, die sich auf Rekonstruktionen konzentriert, großteils Rekonstruktionen verschwundener Bauten, unter anderem auch imaginierte Staatsbauten. In diesen Bilderbüchern sind oft die wesentlichen von den vielen Bauten, die in den neuen Plänen des antiken Rom erscheinen, oder manchmal ganze Ensembles herausgenommen und jeweils so vergrößert, daß sie ein ganzes Blatt füllen. Damit sie in der Vergrößerung gut wirken, sind sie im Detail bereichert und ausgeschmückt. Das erste Werk dieser Art konzipierte Étienne Dupérac auf der Basis von Ligorios und seinem eigenen Romplan (1574-1575). ${ }^{100}$ Das populärste publizierte der römische Stecher Giacomo Lauro ab 1612 unter dem Titel Antiquae urbis Splendor. Vieles ist hier eigenständig erfunden. Dabei hielt sich Lauro nicht etwa genauer als seine Vorgänger an antike Zeugnisse. Seine Rekonstruktionen sind manchmal selbst da sehr freizügig, wo noch Ruinen standen. Eine freie Erfindung bildet auch seine Illustration der Curia Hostilia: ein Haus mit drei Geschossen von Arkaden ungefähr in der Art des Tabularium an den Seiten.

Die Bilderbücher zeigen fast so viele Regierungs- und Verwaltungsbauten wie die berühmten wirklich erhaltenen römischen Monumente. Lauro etwa rekonstruiert zahlreiche Kurien, mehrere Saeptae, Kaiserforen, Basiliken, Palatin, Kapitol etc., alle als stattliche Anlagen. Auf diese Weise werden die früheren Vorstellungen vom alten Rom gründlich korrigiert. Die berühmten erhaltenen Monumente dienten zum größten Teil dem Vergnügen. Es waren Theater, Zirkusse, Stadien, Arenen oder Thermen. Manche von ihnen waren so riesig, daß sie soviel und mehr Zuschauer aufnehmen konnten, wie die größten Städte der Renaissance an Einwohnern zählten. Die Vergnügungen, für die sie dienten, waren meist unkultiviert und grausam. Alberti nahm diesen Aspekt der Antike ziemlich unvoreingenommen hin. Regierungs- und Verwaltungsbauten behandelt er nur kurz, Vergnügungsbauten in der Art der Antike dagegen in aller Ausführlichkeit.

\footnotetext{
98 Frutaz 1962 (note 65), Plan 22.

99 Frutaz 1962 (note 65), Plan 23.

100 Ed. T. Ashby, Rom 1908, und E. Dupérac, Disegni de le ruine di Roma e come anticamente erono. Ed.
}

R. Wittkower, Mailand 1963/1990; H. Zerner, 'Observations on Dupérac and the "Disegni de le ruine di Roma e come anticamente erono"', Art Bulletin 47, 1965, pp. 507-512.101 
Wenn er generell auflistet, was es an Bauten gibt, pflegt er Regierungs- und Verwaltungsbauten zu übergehen. Beispielsweise rät er, in Städten neben Wohnbauten und Straßen Platz zu lassen für Rennplätze, Gärten, Wandelhallen, Schwimmbäder, Zierund Vergnügungsbauten - für Regierungs- und Verwaltungsbauten ist anscheinend kein Platz nötig. ${ }^{101}$ Allerdings stießen die ausschweifenden Vergnügungen und megalomanen Vergnügungsbauten schon in der Antike auf heftige Kritik aus moralischer Warte. So bei berühmten Autoren wie Seneca, Plinius, Cicero, Tacitus und natürlich den Kirchenvätern. Bei aller Bewunderung für die Antike teilten viele Humanisten diese Haltung. Biondo gehörte auch dazu. In diesem Sinn wurde die Bautätigkeit Papst Sixtus' IV. ausdrücklich gegen die Antike abgesetzt, wo eitel Wollust und Prunksucht bestimmend gewesen seien. ${ }^{102}$ Die Bilderbucher des antiken Rom aus der Spätrenaissance korrigierten die alten Vorstellungen offenbar im gleichen Geist und nicht nur aufgrund neuer archäologischer Erkenntnisse. Indem sie die Regierungsbauten vermehrten und als stattliche Gebäude hinstellten, stellten sie einen im Sinn der Renaissance angemesseneren Proporz unter den Monumenten her.

Zudem lebte im Bereich der Pläne und Bilderbücher vom antiken Rom die Vorstellung von der grandiosen Erscheinung des Kapitols wieder auf. Sie verband sich mit neuen archäologischen Erkenntnissen. Aber diese wurden so gedeutet, daß das Kapitol wieder als ein Regierungszentrum erschien. Die Curia Calabra lieferte den Schlüssel dazu (fig. 14). Lauro gibt ihm sogar eine Disposition, die generell Michelangelos Konzeption für die Erneuerung entsprach: mit einem Hof inmitten der Bauten und sogar auf das Marsfeld orientiert bzw. auf die im Mittelalter und in der Renaissance bewohnte Zone, das Abitato.

Wie schön man sich immer das Kapitol vorstellte, es blieb nur ein Regierungssitz unter vielen, die verstreut über die ganze Stadt lagen. In der Renaissance war man aber gewohnt, daß der Sitz der Regierung, auch wenn er auf mehrere Bauten verteilt ist, im Zentrum der Stadt, beim Hauptplatz, liegt. So war es in den mittelalterlichen Städten, und so blieb es bei Neubauten. So wird es empfohlen in allen Architekturtraktaten, die mehr als Alberti auf die reale Situation der eigenen Zeit Rücksicht nehmen, von Filarete, Francesco di Giorgio und Pietro Cataneo (1554/1567). ${ }^{103}$ Daraus erwuchs sogar deutliche Kritik an der Urbanistik im alten Rom. Cataneo führt aus: ${ }^{104}$ Rom, die Herrscherin der Welt, sei zwar bei seiner Gründung klein gewesen und habe nur das Kapitol und den Palatin eingeschlossen, trotzdem hätte man bei der späteren Erweiterung viele öffentlichen Bauten und andere Elemente besser verteilen können, als es ausgeführt wurde. Denn, abgesehen von der Verwinkelung und schlechten Verteilung von Straßen und Plätzen (die bereits Livius, $A b$ Urbe condita 5.55.5 und Tacitus, Annales 15.38,43 ansprechen). hätte ein großer Teil der Gebäude, die weit ab vom Forum Romanum, dem Hauptplatz und Zentrum der Stadt, stehen, statt dessen um oder bei diesem Platz errichtet werden sollen, so besonders die am meisten frequentierten, wie die Basiliken, wo die Magistrate Recht sprachen, und ähnlich einige Kurien und Rostren, die weit vom Forum entfernt gelegen hätten, aber eigentlich bei ihm hätten liegen sollen. Auch hätte man die Comitien, die Volksversammlungen, nicht

101 Alberti, De re aed. IV 3. Theuer, pp. $191.102 \mathrm{H}$. Günther, "'Insana aedificia thermarum nomine extructa". Die Diokletiansthermen in der Sicht der Renaissance', in: Hülle und Fülle. Festschrift für Tilmann Buddensieg, Alfter, 1993, pp. 251-283.

${ }^{102}$ H. Günther, "'Insana aedificia thermarum nomine extructa". Die Diokletiansthermen in der Sicht der
Renaissance', in: Hülle und Fülle. Festschrift für Tilmann Buddensieg, Alfter, 1993, pp. 251-283.

103 Antonio Averlino detto Il Filerete, Trattato di architettura. Ed. A.M. Finoli, L. Grassi, Milano, 1972 , p. 272ff. (Anf. Buch X); F. di Giorgio 1967 (note 29), p. 350; Cataneo 1554 , p. $11 \mathrm{v}$.

104 Cataneo 1554, p. $7 \mathrm{v}$. 


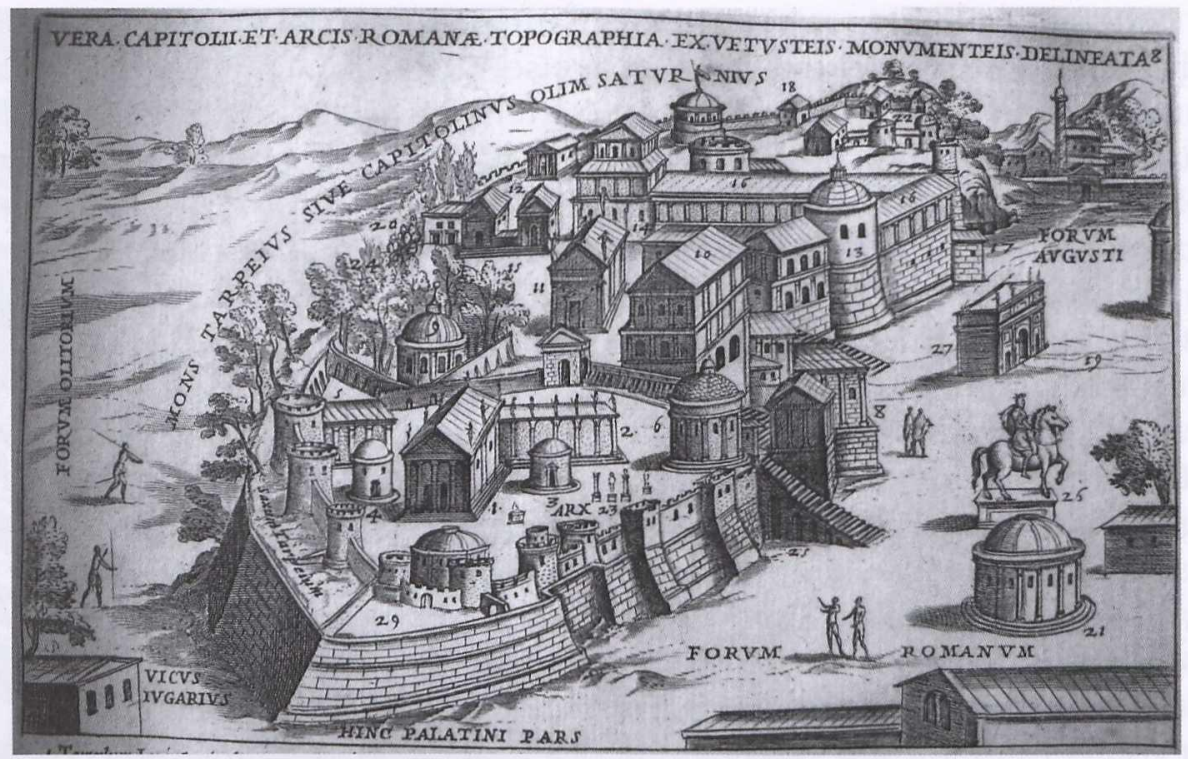

14 Das Kapitol zu Rom nach Giacomo Lauro, Antiquae urbis Romae splendor, Roma, 1612; hinten die Curia Calabra und der Senatulum.

weit draußen auf dem Marsfeld in der Saepta, sondern auf dem Forum oder nahe bei ihm abhalten sollen. Insgesamt hätten sich Volk und Senat in diesen und vielen anderen Orten und Tempeln weitab vom Hauptplatz und Zentrum der Stadt versammelt. Cataneo gibt die Verantwortung für diesen Mißstand den Auguren, weil aus den antiken Schriften bekannt war, daß sie die Versammlungsorte auswählten. Damit wollte er vielleicht im Geist der Rationalität der Renaissance sagen: Das kommt davon, wenn sich die Stadtplanung nach Vogelschauen statt nach vernünftigen Kriterien richtet. Vielleicht führt er die Auguren auch nur an, um anzudeuten, daß solche Unterschiede zwischen antiker und neuer Architektur, wie sie bei den Regierungsbauten auftreten, durch den Wandel der gesellschaftlichen Verhältnisse im Ganzen bedingt sind. Jedenfalls rief der Florentiner Historiker Francesco Guicciardini seinem Gegner Niccolò Machiavelli in Erinnerung:

"Wie täuschen sich diejenigen, die sich mit jedem Wort auf die Römer beziehen. Man müßte einen Staat mit gleichen Lebensbedingungen haben wie sie und regieren wie sie. Aber bei den unterschiedlichen Verhältnissen ist es ebenso unangemessen wie es wäre, wenn ein Esel an einem Pferderennen teilnehmen sollte". ${ }^{105}$

105 "Quantosi ingannanocolorochea ogniparolaallegano e Romani! Bisognerebbe avere una città condizionata come era loro, e poi governarsi secondo quello esemplo: el quale a chi ha le qualità disproporzionate è tanto disproporzionato, quanto sarebbe volere che uno asino facessi el corso di uno cavallo". Francesco Guicciardini, Ricordi, diari, memorie, Ed. M. Spinella, Pordenone, 1991, p. $203 \mathrm{ff}$, Ricordi 110. 